
FOR AERONAUTICS

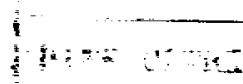

TECHNICAL NOTE 2772

DRIVING STANDING WAVES BY HEAT ADDITION

By Perry L. Blackshear, Jr.

Lewis Flight Propulsion Laboratory Cleveland, Ohio

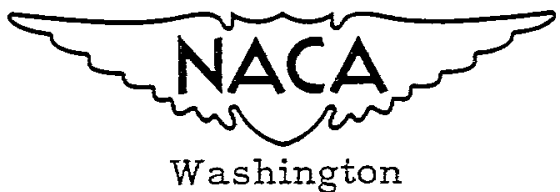

Washington

August 1852

AFMOC 
TECENICAL NOTE 2772

\section{DRIVING STANDING WAVES BY HEAT ADDITION}

By Perry I. Blackshear, Jr.

\section{SUMMARY}

Types of burner instability are enumerated and the role of standing waves in burners is discussed. The status of the problem of flame-driven standing waves is reviewed and a one-dimensional flow theory giving the mechanism whereby a flame drives or damps a standing wave is presented. In this theory, the reflection, transmission, and amplification of waves passing through a flame region were determined from the continuity and momentum equations. For the model considered, waves were found to pass through the flame front with their velocity amplitudes unaltered so long as the Plame area remained unchanged. A change in flame area acted as a source of waves propagating simultaneously into the hot and cold gases on either side of the flame zone.

$$
\text { When the parameter } \frac{1-2 M_{0}}{\sqrt{T_{1} / T_{2}}+2 M_{0}} \text { is unity (where } M_{O} \text { is average }
$$

inlet Mach number and $T_{1} / T_{2}$ the temperature ratio across the rlame zone), these waves are of equal magnitude and the driving criterion is that proposed by Rayleigh, namely, that for heat to drive a standing wave, the heat input should maximize with time at a place where the pressure in the standing wave varies and at a time when the pressure is near its local maximum value.

For $\frac{1-2 M_{0}}{\sqrt{T_{I} / T_{2}}+2 M_{0}}>I$, the flame-generated wave passing into the hot gas dominates and for $\frac{1-2 M_{0}}{\sqrt{\mathbb{P}_{1} / T_{2}}+2 M_{0}}<1$, the flame-generated wave passing into the cold gas dominates. Phase requirements for driving and damping at these conditions are given.

The role played by a flame in a standing-wave system was examined experimentally by measuring the ability of a flame to damp a standing wave. Factors investigated were: fuel-air ratio, inlet gas temperature, sound amplitude, inlet velocity, flame-holder position, and flame area as a function of time. 
The percentage flame-area disturbance was found proportional to percentage velocity disturbance entering the flame zone. The phase lag of the area disturbance behind the velocity disturbance was found dependent upon flame-holder geometry and flame speed; in general, this lag increased as the flame speed decreased.

The flame shape was strongly influenced by a radial component of the time-varying flow which appeared to follow a potential-flow velocity distribution in the neighborhood of the flame holder. This radial component of flow caused an initial growth of the disturbance in the flame front until a position was reached such that normal flame propagation caused these disturbances to undergo an apparent decay.

The one-dimensional theory seems adequately to explain the experimental observations.

\section{INTRODUCTION}

In the field of jet-engine combustor research, burner instabilities are frequently encountered. A brief description of these instabilities is found in the names they have been given: in rockets, chugging and. screaming; in afterburners, rumbling and squealing; in ram jets, rough burning, resonance, pulsing, and whistling; even in Bunsen burners, occasional buzzing.

In most cases a given combustor that exhibits one or more of these instabilities can be modified by a number of trial-and-error methods and the instabilities eliminated within the design range of operating conditions. For example, chugging rockets are quieted by increasing the pressure drop across the injectors; squealing in afterbumers is subdued by increasing the velocity past the flame holders.

The art of combustor design in its present state allows the designer, in most cases, to "cure" a specific burner of instabilities when they arise but can in no way guarantee that a new design will be free of them. Any of these instabilities can be extremely detrimental to engine performance.

Fenn, Forney, and Garmon (reference 1) have identified three types of instability found in ram-jet-type burners. They, along with a number of other investigators, have identified one type as resonance of the standing-wave or organ-pipe type. There is further evidence that this resonance can trigger other forms of instability that involve fuelinjection systems or supersonic diffusers.

In 1859, Rijke (reference 2) discovered a method of driving standing waves by heat addition. Rayleigh (reference 3 ) and subsequently others have postulated an explanation of the Rijke tones (references 4 and 5 ), and have defined a criterion whereby flames (references 6 and 3 ) drive a 
standing wave. In general, this criterion for driving a standing wave by the periodic adoition of heat has been that heat be added at a place where the pressure in the standing wave varies and at a time when the pressure is a maximum.

So far no mechanism whereby this heat addition can drive or damp an oscillation has been described in detail. An attempt to do this is made in the following theory of flame-driven standing waves derived at the NACA Lewis laboratory by employing one-dimensional flow equations to deduce the laws governing the transmission, reflection, and amplification of waves that approach a flame front.

This point of view reveals the important parameters controlling the ability of a flame to drive or to damp an oscillation. The way these parameters vary in an actual flame is examined experimentally by measuring ability of the flame to damp a controlled imposed oscillation.

\section{THEORY OF FTAME-DRIVEN STANDTNG WAVES}

Presuppose the existence of a standing wave and consider the region in a straight duct bounded on each end by a pressure loop at $x=0$ and $x=I$, as shown in figure $I$. (AII symbols are defined in the appendix.) The flame holder is located at $x_{f}$ at which point there is a flame region, assumed to be short compared with $I$. In the region $0 \leq x \leq x_{1}$, the gas is at temperature $T_{1}$ and, in $x_{p} \leq x \leq I$, at temperature $T_{2}$. Assume the wave equation applies to these regions considered separately. The boundary conditions connecting the two regions at $x_{f}$ can now be obtained from the continuity and momentum equations.

Consider the magnified view of the flame zone in figure 2 where the flame is shown anchored on a flame holder, and the zone 1 to 2 is considered incompressible. The velocity entering plane 1 is taken to be

and leaving plane 2 to be

$$
\left.\begin{array}{c}
\mathrm{U}_{1}=\mathrm{U}_{\mathrm{av}}+\mathrm{v}_{1} \\
\mathrm{U}_{2}=\mathrm{U}_{\mathrm{av}} \frac{\rho_{1}}{\rho_{2}}+\mathrm{v}_{2}
\end{array}\right\}
$$

The instantaneous flame area is

$$
A_{I}=A_{a v}+a
$$


so that

$$
U_{a v} A=u_{\rho} A_{a v}
$$

where $u_{f}$ is flame speed; $U_{a v}, U_{a v} \rho_{1} / \rho_{2}, A_{a v}$ are mean values; $v_{1}$, $v_{2}$, and $a$ are perturbations; and $A$ is the cross-sectional area of the duct.

Since the region between $I$ and 2 is incompressible,

and

$$
\left.\begin{array}{c}
\mathrm{AU}_{1}=\mathrm{A}_{f} U_{f}+\frac{\partial V_{f}}{\partial t} \\
\mathrm{AU}_{2}=\mathrm{A}_{f} U_{f} \frac{\rho_{I}}{\rho_{2}}+\frac{\partial V_{f}}{\partial t}
\end{array}\right\}
$$

where $V_{f}$ is the volume of cold gas enveloped by the flame.

From equations (1) to (4),

$$
v_{2}=v_{1}+a \frac{u}{A_{a v}}\left(\frac{\rho_{1}}{\rho_{2}}-1\right)
$$

In order to obtain the pressure relation across the flame zone, consider the one-dimensional momentum equation for inviscid incompressible flow:

$$
\begin{aligned}
P_{1}-P_{2}= & \rho_{2} U_{2}^{2}-\rho_{1} U_{1}^{2}+\frac{I}{A} \int \frac{\partial \rho U}{\partial t} d V \\
= & \rho_{2} U_{2}^{2}-\rho_{1} U_{1}^{2}+\frac{I}{A} \frac{\partial}{\partial t}\left(\rho_{1} U_{1} V_{f}+\rho_{2} U_{2} V_{h}\right) \\
= & \rho_{2} U^{2}-\rho_{1} U_{1}^{2}+\frac{1}{A}\left(\rho_{1} U_{I} \frac{\partial V_{f}}{\partial t}-\rho_{2} U_{2} \frac{\partial V_{f}}{\partial t}\right)+ \\
& \frac{I}{A}\left(V_{f} \frac{\partial \rho_{1} U_{1}}{\partial t}+V_{h} \frac{\partial \rho_{2} U_{2}}{\partial t}\right)
\end{aligned}
$$


Where $V_{h}$ is the volume between planes $I$ and 2 having density $\rho_{2}$ and

$$
V_{h}=\left(x_{2}-x_{1}\right) A-V_{f}
$$

But from equations (1) to (4)

$$
\frac{\partial v_{f}}{A \partial t}=-\frac{\partial v_{h}}{A \partial t}=v_{1}-\frac{a U_{a V}}{A_{a V}}
$$

Inserting equations (1) to (5) and (7) in equation (6) and neglecting squares of perturbations gives the following expression for equation (6):

$$
P_{1}-P_{2}=\rho_{1}\left(\frac{\rho_{1}}{\rho}-1\right) U_{a v}{ }^{2}\left(I+\frac{2 a}{A_{a v}}\right)+\frac{I}{A}\left(v_{f} \frac{\partial \rho_{1} U_{I}}{\partial t}+V_{h} \frac{\partial \rho_{2} U_{2}}{\partial t}\right)
$$

Now

$$
\frac{1}{A}\left(V_{I} \frac{\partial \rho_{1} U_{I}}{\partial t}+V_{h} \frac{\partial \rho_{2} U_{2}}{\partial t}\right)
$$

can be shown from reference 7 to be approximately equal to

$$
\Delta x_{1}\left(-\frac{\partial p_{1}}{\partial x}-U_{1} \frac{\partial \rho_{1} U_{1}}{\partial x}\right)+\Delta x_{2}\left(-\frac{\partial p_{2}}{\partial x}-U_{2} \frac{\partial \rho_{2} U_{2}}{\partial x}\right)
$$

These terms represent variously the gradients in pressure and momentum in the cold and hot portions of the flame zone which have been assumed short compared with wavelength and may be neglected.

Outside the flame zone the wave equation applies. For the cold region $\left(0 \leq x \leq x_{\hat{I}}\right)$ considered separately (reference 7$)$,

$$
\frac{\partial^{2} \xi_{1}}{\partial t^{2}}=c_{1}^{2} \frac{\partial^{2} \xi_{1}}{\partial x^{2}}
$$

the complete solution for which is

$$
\xi_{1}=\overrightarrow{F k}\left(\frac{x}{c_{1}}-t\right)+\stackrel{t}{s k}\left(\frac{x}{c_{1}}+t\right)
$$

This is for no flow, where $\xi$ is particle displacement, $c_{1}$ is speed of sound in $0 \leq x<x_{f}$, and $k$ is frequency. 
The terms $\vec{F}$ and $\leftarrow$ are identified as right-and left-traveling waves, respectively, propagating at the speed of sound $c_{1}$. For the case with flow, the right propagation velocity is $c_{1}+U_{a v}$ and the left propagation velocity is $c_{1}-U_{a v} ; \xi_{1}$ becomes

$$
\xi_{I}=F\left[k\left(\frac{x}{c_{1}+U_{a v}}-t\right)\right]+f\left[k\left(\frac{x}{c_{1}-U_{a v}}+t\right)\right]
$$

Expanding

$$
\frac{x}{c_{I}+U_{a v}}=\frac{x}{c_{1}}\left(1-M_{0}+M_{0}^{2} \cdot \cdots\right)
$$

and

$$
\frac{x}{c_{1}-U_{a v}}=\frac{x}{c_{1}}\left(1+M_{0}+M_{0}^{2}+\cdots \cdot\right)
$$

where $M_{0}=\frac{U_{a v}}{c_{1}}=$ inlet Mach number. Defining

$$
t^{\prime}=t+M_{0} \frac{x}{c}
$$

The general solutions may then be approximated by

$$
\xi_{1}=F\left[k\left(\frac{x}{c_{1}}-t^{i}\right)\right]+f\left[k\left(\frac{x}{c_{1}}+t^{i}\right)\right]
$$

Therefore, for moderate Mach numbers the succeeding arguments for $t$ apply equally well for $t^{\top}$. (Since $M_{0} \frac{x}{c_{1}} \approx \frac{U_{a v} \frac{\rho_{1}}{\rho_{2}}}{c_{2}} \frac{x}{c_{2}}$, the same $t^{\prime}$ is used in $x_{f} \leq x \leq I_{\text {. }}$ )

For small perturbations that satisfy the boundary condition at $x=0$, namely, $\xi_{1}=0$, the particular solutions may be written

$$
\xi_{1}=B_{1} \overrightarrow{\sin k}\left(\frac{x}{c_{I}}-t\right)+B_{1} \stackrel{\leftrightarrow}{\sin k}\left(\frac{x}{c_{1}}+t\right)=2 B_{1} \cos k t \sin \frac{k}{c_{I}} x
$$


where $B_{1}$ is ampititude.

The following equations are employed from reference 7:

$$
p_{1}=-c_{1}^{2} \rho_{1} \frac{\partial \xi_{1}}{\partial x}
$$

and

$$
v_{1}=\frac{\partial \xi}{\partial t}
$$

where $P_{I}$ and $v_{I}$ are periurbation pressures and velocities for this region and it is noted that for a right-traveling wave

$$
\begin{aligned}
& \overrightarrow{p_{1}}=\rho_{1} c_{1} \overrightarrow{v_{1}} \\
& \overrightarrow{p_{2}}=\rho_{2} c_{2} \overrightarrow{v_{2}}
\end{aligned}
$$

and a left-traveling wave

$$
\}
$$

Similar relations are derived for $x_{p}<x \leq I$ such that the boundary condition at $I$ is satisfied, again $\xi_{2}=0$.

The boundary conditions at $\mathrm{x}_{\mathrm{f}}$ state that

$$
\begin{gathered}
v_{2}=v_{1}+\frac{a}{A_{a v}} U_{a v}\left(\frac{\rho_{1}}{\rho_{2}}-1\right) \\
P_{1}-P_{2}=\rho_{1}\left(\frac{\rho_{1}}{\rho_{2}}-1\right) U_{a v} 2\left(1+\frac{2 a}{A_{a v}}\right)
\end{gathered}
$$

Let

$$
\frac{a}{A_{a v}} U_{a v}\left(\frac{\rho_{1}}{\rho_{2}}-1\right)=a^{\prime}
$$

such that equation (5) becomes $v_{2}=v_{1}+a^{\prime}$ and equation (8) becomes 


$$
P_{1}-P_{2}=\rho_{1}\left(\frac{\rho_{1}}{\rho_{2}}-1\right) U_{a v}^{2}+2 \rho_{1} U_{a v} a^{\prime}
$$

These equations are interpreted as follows. When $a^{\prime}$ is zero, waves pass through the flame zone with their velocity components unaltered. The pressure components in this case are then determined by relations (12).

When $a^{\prime}$ is not zero, waves are generated at the flame front traveling into the hot and cold gases. The net change in velocity across the flame holder caused by these waves is equal to

$$
a^{\prime}=\overleftrightarrow{a_{\eta}^{\prime}}+\overrightarrow{a_{r}}
$$

and a net change in pressure caused by these waves is equal to

$$
2 \rho_{1} U_{a v} a^{\prime}=-\rho_{1} c_{1} \overleftrightarrow{a_{l}}-\rho_{2} c_{2} \overrightarrow{a_{r}}
$$

or, solving for $\overrightarrow{a_{r}}$ and $\overleftarrow{a_{\eta}}$,

$$
a_{r}^{\prime}=a^{\prime}\left(\frac{1-2 M_{0}}{1+\frac{\rho_{2} c_{2}}{\rho_{1} c_{1}}}\right) \approx \frac{1-2 M_{0}}{1+\sqrt{\frac{T_{1}}{T_{2}}}}
$$

where $\mathrm{M}_{O}=\frac{\mathrm{U}_{\mathrm{av}}}{\mathrm{C}_{I}}=$ iniet Mach number and

$$
a_{1}^{\prime}=-a^{\prime}\left(\frac{\frac{\rho_{2} c_{2}}{\rho_{1} c_{1}}+2 M_{0}}{1+\frac{\rho_{2} c_{2}}{\rho_{1} c_{1}}}\right) \approx-a^{\prime} \frac{\sqrt{\frac{T_{1}}{T_{2}}}+2 M_{0}}{1+\sqrt{\frac{T_{1}}{T_{2}}}}
$$

where $a_{p}{ }^{\prime}$ and $a_{q}{ }^{\prime}$ are the right- and left-traveling flame-generated waves. The ratio of magnitudes of these waves is

$$
-\frac{a_{r}{ }^{\prime}}{a^{\prime}}=\frac{I-2 M_{O}}{\frac{\rho_{2} c_{2}}{P_{1} c_{I}}+2 M_{O}} \approx \frac{1-2 M_{O}}{\sqrt{\frac{T_{I}}{T_{2}}+2 M_{0}}}
$$

If this parameter is greater than 1 , the right-traveling wave will dominate; when it is less than 1 , the left-traveling wave will dominate. 
If the net effect is such that flame-generated waves are phased with the corresponding right- and left-traveling components of the standing wave, the flame will tend to drive the oscillation; when they are $180^{\circ}$ out of phase, the flame will damp the oscillation. The net velocity $v_{I}$ causes the disturbances in flame area. The phase lag of $a^{\prime}$ behind $v_{1}$ where maximum driving or damping occurs is therefore of interest.

For $v_{1}=-2 B k \sin k t \sin k_{f} / c_{1}$, let

$$
a^{\prime}=- \text { E } \sin k(t-\varphi)
$$

Where $F$ is a proportionality factor.

But the right-traveling component of $v_{1}$ is

$$
\overrightarrow{v_{1}}=-B k \cos k\left(\frac{x_{f}}{c}-t\right)=-B k \sin \left[k t-\left(\frac{3}{2} \pi+\frac{k x_{f}}{c}\right)\right]
$$

or for $a^{\prime}$ to be in phase with $\overrightarrow{v_{1}}$,

$$
\begin{aligned}
& \varphi=\frac{3}{2} \frac{\pi}{k}+\frac{x}{c} \\
& k=\frac{2 \pi}{\tau} \\
& \varphi=\frac{3}{4} \tau+\frac{x}{c}
\end{aligned}
$$

Similarly, for a' to be in phase with $\overleftarrow{v_{I}}$,

$$
\varphi=\frac{3}{4} \tau-\frac{x_{f}}{c_{I}}
$$

Obviously, $a^{\prime}$ can supply right and left travelers simultaneously in phase with $\vec{v}_{I}$ and $\overleftarrow{v}_{I}$ at $x_{f}=0$ or $x_{f}=\lambda / 2$ only. For any value of $x_{f}$ and $\varphi$, the relative ability to drive for a given $a^{\prime}$ is

$$
D_{a^{i}=c}=\frac{I-R}{I+R}\left(\cos k \varphi \sin \frac{k x_{I}}{c_{I}}\right)-\sin k \varphi \cos \frac{k x_{I}}{c_{I}}
$$


where

$$
\frac{1}{R}=\frac{1-2 M_{0}}{\frac{\rho_{2} c_{2}}{\rho_{1} c_{1}}+2 M_{0}} \approx \frac{1-2 M_{0}}{\sqrt{\frac{T_{1}}{T_{2}}}+2 M_{0}}
$$

and for $a^{\prime} \propto v_{I}$

$$
D_{a^{\prime}} \omega v_{1}=\sin \frac{k x_{f}}{c_{I}} D_{a^{\prime}=c}
$$

where negative values of $D$ imply damping, and $D$ is driving effectiveness. Maximum driving or damping occurs where

$$
\varphi=\frac{1}{k} \tan ^{-1}\left(\frac{I+R}{I-\bar{R}} \frac{I}{\tan \frac{k x_{f}}{c_{I}}}\right)
$$

A plot of phase lags for maximum driving and maximum damping, respectively, $\varphi_{+}$and $\varphi_{-}$, in fractions of $\tau$ as a function of $x_{f}$ in fractions of $\lambda_{1}$ is given in figure 3 for values of

$$
\frac{1-2 M_{0}}{\sqrt{T_{1} / T_{2}}+2 M_{0}}=\frac{1}{2}, 1 \text {, and } 2
$$

In addition, the envelope of constant $D_{a^{\prime}}, D_{a^{\prime}} \infty \mathrm{v}^{\prime}$, and a few representative values of $D_{a}, \infty v^{\prime}$ for a few arbitrary values of $\varphi$ are plotted against $x_{f}$.

In figure 4 relative driving effectiveness is plotted against $\varphi$ for several values of $x_{f}$.

The driving criterion of Rayleigh, then, is for the case of

$$
\frac{1-2 M_{0}}{\sqrt{T_{1} / T_{2}}+2 M_{0}}=1
$$

since for this condition, $\varphi_{+}$requires heat release to occur when pressure is a maximum.

The experiments Rayleigh correlated with this criterion were for low Mo and $\sqrt{T_{1} / T_{2}} \approx 1$. 
Other properties of the resonating system may be deduced. For $\varphi$ where maximum driving or damping occurs, the period of oscillation will be

$$
\tau=\frac{2 x_{I}}{c_{1}\left(I-M_{0}^{2}\right)}+\frac{2\left(I-x_{I}\right)}{c_{2}\left[I-\left(\frac{\rho_{1} U_{a v}}{\rho_{2} c_{2}}\right)^{2}\right]}
$$

or

$$
\tau \approx \frac{2 x_{f}}{c_{1}\left(I-M_{0}^{2}\right)}+\frac{2\left(I-x_{f}\right) \sqrt{\rho_{2} / \rho_{I}}}{c_{1}\left(I-M_{0}^{2} \rho_{1} / \rho_{2}\right)}
$$

For $\varphi$ other than $\varphi_{+}$or $\varphi_{-}$, the flame-generated waves will alter the wave form and the frequency. This frequency shift is dependent upon the departure of $\varphi$ from $\varphi_{+}$and upon the ratio of the amplitudes of the flame-generated waves to the existing standing waves. For any value of $\varphi$ and for $a^{\prime} D \leq B$, the frequency of resonance is

$$
f=\frac{1-\left(\frac{I}{4 \pi} \sin ^{-1} \frac{a^{3} D}{B}\right) \sin k\left(\varphi_{+}-\varphi\right)}{2 x_{f}} \frac{2\left(I-x_{f}\right.}{c_{1}\left(I-M_{0}^{2}\right)}+\frac{c_{2}\left[1-\left(\frac{\rho_{1} U_{2 V}}{\rho_{2} c_{2}}\right)^{2}\right]}{c_{2}[}
$$

(where $D$ is maximum possible effectiveness at a given $x_{f}$ ). Plots of relative driving effectiveness and relative frequency are shown in figure 5 plotted against $\varphi$. These plots apply for any flame-holder position $x_{\hat{f}}$ and for any value of $R$.

\section{Interpretation}

Before the experimental investigation is discussed it might be advisable to give a resume of the physical implications of the theory.

For the model considered, waves can pass through the flame zone with their velocity components unaltered, providing that the flame area remains unchanged. For this case there is no driving or damping. If the flame area changes, the flame can drive or damp depending on the phase lag between the gas velocity and flame-area perturbations and the magnitude of the area perturbation, as shown in figure 3 . The driving 
effectiveness given in figure 3 gives an index to the increase in wave amplitude each time it passes through the flame zone. If this increase in amplitude exactly compensates the loss per cycle to nonflame damping, the system will resonate. If this increase is greater or less than that required to compensate for losses the amplitude will increase or decrease, respectively, until flame blow-out occurs or an equilibrium is reached.

It was assumed that the flame zone was short compared with the quarter wavelength. In a duct it may be possible to excite the first several modes of vibration without violating this assumption. In this case the region of interest is the half-wavelength segment between the two pressure loops bracketing the flame zone, and figure 3 applies to this region.

The mode of vibration the flame will select to drive will probably depend upon sound intensity and will probably be the one for which the ratio of flame amplification to duct damping is the greatest.

Brief mention should be made concerning the point at which a flamedriven standing wave would give way to another type of oscillation. In brief, the standing wave should exist where flame amplification and subsequent attenuation per cycle is small. The point where this ceases to apply would be in the region where the attenuation at one end of the resonating system is such that the strength of the reflected wave equals the strength of the flame-generated wave alone. On the far side of this point, anticipated resonance is of a type having an acoustic length either two or four times the length from flame to reflecting end (as opposed to attenuating end) and a frequency dependent upon this acoustic length and upon the time lag between disturbance (velocity or pressure) and resulting flame-generated wave.

\section{EXPERIMENTAL INVESTIGATION}

It was felt that far more control could be exerted in examining experimentally the effect of a flame on a standing-wave system by measuring the ability of the flame to damp an imposed standing wave than in examining spontaneous excitations. This was accomplished with the following system:

Apparatus. - A metered homogeneous mixture of propane and air was fed to a l-inch-aiameter burner as shown in figure 6 , through a variablearea pintle-type nozzle. Standing waves in this burner were driven by a piston speaker through a high-impedance source tube (reference 8 ) at the base of the bumer. Sound level was measured in the burner base by two flush-mounted button microphones. The flame holders employed (fig. 7) could be positioned in a test section where desired. Auxiliary equipment included a hot-wire anemometer, a stroboscope, a camera, and a photocell. 
Froperties of system. - The Reynolds number in the test section was about 500. The velocity profile with sound appeared to be a superposition of a potential-flow profile for the time-varying flow upon the steady laminar flow (fig. 8) with no flame holder present. The time-varying velocity at points across the test section is indicated by the numbered inserts, showing that near the walls flow reversal occurred. Placing a flame holder in the test section gave velocity profiles shown in figure 9. A comparison of the time-varying component with a computed (reference 9) two-dimensional potential flow in figure lo shows that fair agreement exists except for a boundary layer. This boundary-layer thickness seemed to decrease with an increase in Reynolds number of the steady flow.

A variety of mufflers were examined and one was selected having a fairly constant attenuation for the first four modes of vibration.

As the pressure drop across the inlet orifice increased, the random noise increased while the damping coefficient of the burner decreased until a pressure drop of 1.5 inches of mercury was reached. Above this value, the noise continued to increase but the damping coefficient remained substantially constant.

A sample of the spontaneously excited frequencies found in the initial exploratory work is shown in figure 11. Here a 64-mesh-per-inch screen was used as a flame holder. The exhaust end of the tube was alternately open or masked by a plate containing a l/l6-inch-diameter orifice. With this arrangement, that mode of vibration was excited such that the flame holder was upstream of a pressure loop, with the tube acting as either a closed-closed $(\lambda / 2)$ or closed-open $(\lambda / 4)$ resonator.

Procedure. - Two indexes of damping coefficient were employed (reference 10): the width of resonant peak, or the voltage required to drive the speaker for a constant sound intensity in the burner. The role of the flame in damping the imposed oscillation was investigated by considering the following variables: fuel-air ratio, inlet temperature, flameholder position, sound amplitude, and inlet velocity.

Effect of fuel-air ratio on damping. - The efiect of fuel-air ratio on damping is illustrated in figures $12(\mathrm{a})$ to $12(\mathrm{c})$, for the first four modes of vibration with all three flame holders. The curves seem to be, within limits, symmetrical about the fuel-air ratio of maximum flame speed, 0.07 (reference 1I). That this would indicate a strong dependence on flame speed is not immediately evident since flame-speed studies (references 11 and 12) show flame speeds as asymmetrical about a fuel-air ratio of 0.07 .

However, these references do not agree on flame speeds for rich mixtures; reference 12 indicates higher and reference 11 lower values than 
those required for symmetry. A few check readings of photographs of flames burning on the one-hole flame holder within the tube showed symmetry about a fuel-air ratio of 0.07 and agreed moderately well with the reference lean values.

In figure $12(\bar{d})$ the voltage to speaker and the observed frequency at resonance for the third mode of vibration are plotted against fuel-air ratio. For the three- and five-hole flame holders, these data show good agreement with figure 5 if fuel-air ratio is taken as an index of $\varphi$ such that $\varphi$ increases as flame speed decreases.

This is interpreted to mean that the phase lag of the flame-area change behind the velocity perturbation has a strong flame-speed dependence. A comparison of figures $12(a),(b)$, and (c) indicates a strong dependence on flame-holder geometry.

Data shown in figure 12 were taken in a steel test section. Some of the heat transferred from flame to wall downstream of the flame holder was conducted upstream and thus preheated the combustible mixture. Therefore, when these figures are compared with subsequent curves taken in glass tubes, this preheating must be considerea.

Effect of inlet temperature on damping. - The effect of fuel-air ratio on damping is shown in figure 13 for various gas temperatures. The fuel-air ratio at which damping peaked for each temperature was such that flame speed remained the same. If a/Aav is assumed proportional to $v_{1} / U_{a v}$, and sound intensity is assumed constant $\left(v_{I} \infty T_{1}^{\frac{1}{4}}\right)$, then

$$
a^{\prime}=\frac{A}{A_{a v}} U_{a v}\left(\frac{\rho_{1}}{\rho_{2}}-1\right) \infty v_{1} \propto T_{1}^{\frac{1}{4}}
$$

A slight increase in the driving or the damping as temperature increases would therefore be expected for a constant mass flow, as indicated in figure 12 .

Effect of flame-holder position on damping. - The effect of flameholder position $y$ on damping coefficients for the third mode of vibration is shown in figure 14. It has been shown from theoretical considerations that the damping would be expected to depend upon a/Aav, hence $v_{1}$, and upon the phase lag $\varphi$. For the third mode a marked peak is obtained in the curve of damping against fuel-air ratio curve for each position except at $\mathrm{y}=0$. At $\mathrm{y}=0$, the right-traveling velocity wave will not be reflected, hence will not be able to drive or damp, and 
the left-traveling flame-generated wave will be weaker because of a diminished momentum pressure drop. In the range erom $\mathrm{y}=2$ to $y=8 \frac{1}{4}$ inches, the magnitude of flame damping increased and the fuel-air ratio where maximum damping occurred shifted toward the fuel-air ratio of maximum flame speed. This effect would imply from theory that phase lag diminishes for the third mode as flame speed increases, as is demonstrated subsequently. Maximum damping would be expected to occur at $y=\lambda / 8 \approx 10$ inches for this mode. The spontaneous excitation of a higher harmonic at $y>8 \frac{1}{4}$ inches precluded verification of this maximum point.

Effect of sound amplitude on damping. - Over the range of amplitude examined ( 120 to $160 \mathrm{db}$ ), there was no perceptible change in the damping characteristics of the Plame, as long as the flame remained seated. This would indicate that the flame component of damping varied directly as the disturbance velocity, that is, a/Aav $\infty v_{1} / \mathrm{U}_{a v}$. Furthermore, it would imply that amplitude did not alter the phase lag. Too large an amplitude would cause the flame to lift or flash back. Tests run at amplitudes that were not large compared with the background noise were impossible to interpret with the equipment employed. A number of strange effects were noted. For example, with a combustible mixture flowing through the tube, a flame ignited at the open end would burn upstream to within an inch of the flame holder and hover there, exciting spontaneously the first mode of vibration. Imposing the second- or third-mode frequency at a high amplitude would cause the flame to drop down to the flame holder, where it would seat and burn quietly after removal of the imposed vibration.

Effect of inlet velocity on damping. - Damping against fuel-air ratio for different velocities is shown in figure 15 for the three-hole flame holder. The magnitude of damping is seen to vary directly as $\mathrm{U}_{a v}$, whereas the curve shape remains essentially unaltered. This is interpreted as meaning the phase lag is essentially unaltered by flow velocity.

$$
\text { If } a / A_{a v} \text { were proportional to } v_{l} / U_{a v}, \quad a^{\prime}=\frac{a}{A_{a v}} U_{a v}\left(\frac{\rho_{l}}{\rho_{2}}-1\right)
$$
would remain constant as $U_{a v}$ is varied. The apparent increase in $a^{r}$ with an increase in $U_{a v}$ is believed due in part to the Reynolds number effect of the steady flow upon the time-varying component of flow mentioned earlier. The term $a / A_{a v}$, then, is more nearly proportional to $\mathrm{v}_{I} / \mathrm{U}_{\mathrm{av}}{ }^{\mathrm{n}}$, where $\mathrm{n}$ in this instance is slightly less than unity. 
Flame area as a function of time. - Figure 16 shows sample photographs of periodically varying flames at different phases of velocity for the first and third modes of vibration. Here the radial diatortion of the flame at the flame holder due to the radial component of the timevarying potential flow is apparent in the photographs of the third mode of vibration. A particle of gas near the flame-holder rim is given a radial momentum when it passes through the flame holder during outflow which it never loses in the ensuing inflow part of the cycle. This causes an apparent growth in the disturbance when the flame-propagation rate is less than this outflow rate. Because of normal flame propagation, the flame eventually becomes sharply convex toward the burnea gas at these outermost loci and then propagates at a rate that is locally greater than normal laminar flame speed. This gives the appearance of a flame-front disturbance, the amplitude of which is reduced from this height to the tip. The rapid transition to cycloidal cusps in the flame front appeared to be due solely to normal flame propagation in lean and rich flames, and not to flame-front instability (references 14 and I5). That the disturbances did not grow as they have been shown to do in turbulent flames (references 13 and 16 ) would indicate that $a / A_{a v}$, as observed here, is a lower limit.

The first-mode photographs gave evidence of a different flow field. When the amplitude became sufficiently great, the flow appeared conposed of successive ring vortices detached from the flame holder and disturbed the flame in so doing. The transition from this vortex flow to the potential flow of the third mode seemed to occur in the neighborhood of 200 cycles per second for the conditions studied.

Photographic sequences for the three-hole flame holder were taken over a range of fuel-air ratios bracketing the fuel-air ratio where damping maximized.

Damping for these sequences and the frequency variations are shown in figure 17. Figure 18 shows relative instantaneous flame areas of these sequences plotted against degrees behind maximum velocity, giving a measurable lag of flame area, hence heat release, behind velocity. Maximum damping occurred at a fuel-air ratio of 0.0775 . The phase lag for this fuel-air ratio is in the neighborhood of $130^{\circ}$. This is the phase lag which would be predicted from the theory for

$$
\frac{1-2 M_{0}}{\sqrt{T_{1} / T_{2}}+2 M_{0}} \approx 2.2
$$

and for $x_{f}=3 \lambda_{I} / 16$, which seems to fit the conditions imposed for this sequence. In addition, the phase lag increased as flame speed decreased so that the frequency shift is as predicted in figure 5 . 
For the three conditions studied photographically, the phase lag decreased with increasing $U_{f}$ for the third mode, and increased, then decreased with increasing $U_{f}$ for the first mode. No explanation for this devious coupling between flame area and velocity is immediately obvious.

Sumary of experimental results. - From the preceding examinations, a fairly clear picture of the relation between an inlet velocity perturbation and the resultant flame-area perturbation may be obtained. For the third mode and three-hole flame holder, for example, the magnitude of the area disturbance $a / A_{a v}$ is proportional to $v_{I} / \mathrm{J}_{a v}{ }^{n}$ where $n$ is slightly less than unity because of a boundary-layer effect. The phase lag of $a_{I} / A_{a v}$ behind $v_{I}$ depends only upon flame speed $U_{f}$ and not upon $\nabla_{I}$; this phase lag increases with diminishing flame speed. With these properties in mind, it is seen that the agreement with theory is quite good. Disparity exists between theory derived herein and the Rayleigh criterion when $\frac{1-2 M_{0}}{\sqrt{T_{I} / T_{2}}+2 M_{0}} \neq 1$. The shortcomings of the Rayleigh criterion can be verified by experiment: (I) by obtaining a measurable phase lag where maximum damping occurs; (2) by showing a change in the apparent phase lag with $x_{f}$; or (3) by a measured existence of driving or damping at a velocity loop. The experimental verification of these three phenomena is discussed in the following paragraph.

In figure 18 the measured phase lag corresponding to maximum drive is seen to be approximately $130^{\circ}$, which would correspond to $\frac{1-2 M_{0}}{\sqrt{T_{1} / T_{2}}+2 M_{0}} \approx 2.2$ and $x_{f} \approx \frac{3 \lambda}{16}$. According to the Rayleigh criterion, this phase lag could be only $90^{\circ}$ in the region $0 \geq x_{f} \leq \frac{\lambda I}{4}$. These data are not wholly reliable since the time-varying velocity is deduced from the flame slope at the base and would not account for a lag in the jet boundary. Subsequent checks made with a photocell and a hot-wire anemometer coupled to a phase meter verified the lags obtained photographically.

A plot of volts to speaker as an index of damping against fuel-air ratio as an index of phase lag for different flame-holder positions (fig. 14) shows a shift in the phase lag where damping maximizes that is consistent with the theory for the case of $\frac{I-2 M_{0}}{\sqrt{T_{1} / T_{2}}+2 M_{0}}>I$ and could 
not be explained by the Rayleigh criterion. Finally, cases of the spontaneous excitation of the fundamental mode of the duct were recorded with the flame seated at the open end of the duct.

\section{CONCLUSIONS}

The one-dimensional analysis seems an adequate explanation of the mechanism whereby a flame drives or damps a standing wave. This mechanism is as follows: Waves pass through the flame front with their velocity amplitude unaltered. When the net velocity causes a change in flame area, waves are propagated simultaneously into the hot and cold gases. When these flame-generated waves are phased with the existing components of the standing waves, they drive the standing wave; when opposed, they damp.

When the parameter $\frac{I-2 M_{0}}{\sqrt{T_{I} / T_{2}}+2 M_{0}}$ is unity (where $M_{O}$ is average inlet Mach number and $T_{1} / T_{2}$ the temperature ratio across the flame zone), these waves are of equal magnitude and the driving criterion is that proposea by Rayleigh, namely, that for heat to drive a standing wave, the heat input should maximize with time at a place where the pressure in the standing wave varies and at a time when the pressure is near its local maximum value.

The strength of the wave depends largely on the relative magnitude of the disturbance of the flame area a/Aav and the phase relation between $a / A_{a v}$ and perturbation velocity $v_{1}$. The magnitude of $a / A_{a v}$ was found to depend upon $v_{1}$ and average flow velocity in the cold gas $\mathrm{U}_{\mathrm{av}}$ and in most cases varied directly as $\mathrm{v}_{I} / \mathrm{U}_{a v}$. The existence of radial flow in the time-varying component of the flow through the flame holders caused a greater perturbation than would be anticipated in onedimensional flow. The phasing of a/A with $v_{1}$ was found to depend primarily on flame speed and, for the conditions studied, appeared independent of the magnitude of $v_{l} / U_{a v}$. In most cases, the phase lag of $a / A_{a v}$ behind $v_{l} / U_{a v}$ increased as the flame speed decreased.

Lewis Flight Propulsion Laboratory

National Advisory Committee for Aeronautics

Cleveland, Ohio, May 13, 1952 


\section{APPENDIX - SYMBOLS}

The following symbols are used in this report:

A duct cross sectional area, sq ft

Af instantaneous flame area, sq ft

$A_{a v}$ average flame area, sq ft

a $\quad A_{f}-A_{a v}$

$a^{\prime} \quad \frac{a}{A_{a v}} U_{a v}\left(\frac{\rho_{1}}{\rho_{2}}-1\right), f t / \sec$

$a_{\eta}$ left-traveling component of flame-generated ware, ft/sec

$a_{r^{\prime}}$ right-traveling component of flame-generated wave, ft/sec

B amplitude of one component of a standing wave, ft

c velocity of sound, ft/sec

D driving effectiveness, dimensionless

E Voltage required to arive speaker, volts

$F, f$ arbitrary functions

$g$ gravitational constant, $\mathrm{ft} / \mathrm{sec}^{2}$

$\mathrm{k}$ frequency $=2 \pi / \tau$, radians $/ \mathrm{sec}$

I distance between pressure loops $=\lambda / 2$, ft

$\mathrm{M}_{0} \quad \frac{\mathrm{U}_{\mathrm{av}}}{\mathrm{c}_{1}}=$ inlet Mach number

n integer 1,2 , . .

$\mathrm{P} \quad$ instantaneous static pressure $=\mathrm{P}_{\mathrm{aV}}+\mathrm{p}$, Ib/sq ft

$P_{\text {av }}$ average static pressure, $\mathrm{Ib} / \mathrm{sq}$ it

p static pressure of standing wave, $\mathrm{lb} / \mathrm{sq}$ It 


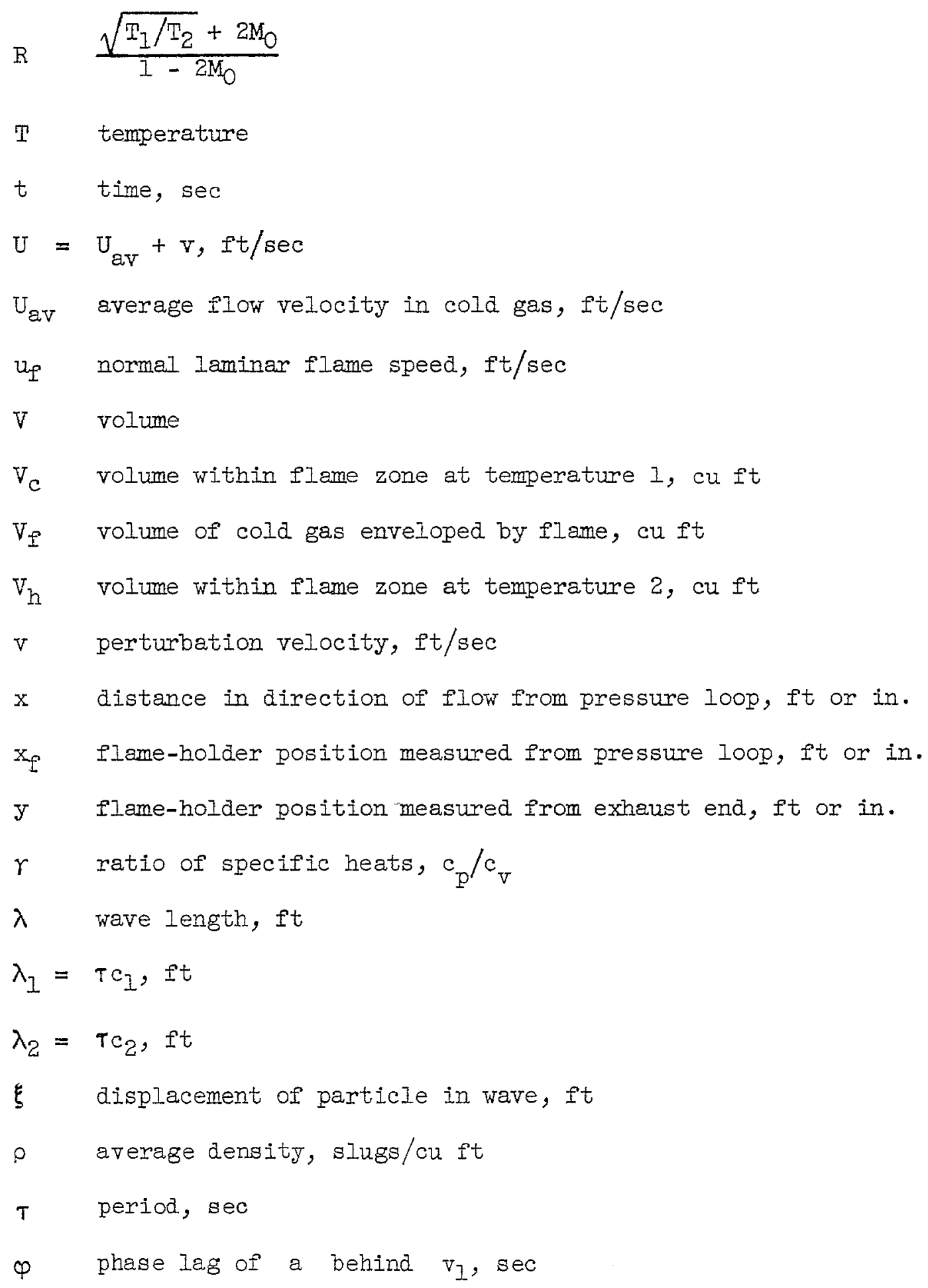


Q phase lag for maximum drive, sec

Q_ phase lag for maximum damping, sec

Subscripts:

1 inlet to flame zone

2 outlet to flame zone

\section{REF'ERENCES}

I. Fenn, J.B., Forney, H. B., and Garmon, R. C.: Burners for supersonic Ram-jets. Ind. and Fng. Chem., vol. 43, no. 7, July 1951, pp. 16631671 .

2. Rijke, P. I.: Notice of a New Method of Causing a Vibration of the Air Contained in a Tube Open at Both Ends. Phil. Mag., ser. 4, vol. XVII, 1859, pp. 419-422.

3. Rayleigh: Theory of Sound. Vol. II, Dover Press, (N. Y.), 1945, p. 226 .

4. Von Iehmann, K. O.: Über die Theorie der Netztöne. Analen der Physik, Bond 29, Heft 6, July 1937, pp. 527-555.

5. Shaw, R. P., and̄ Neuringer, J. I.: Large Amplitude Gas Vibration Source. Project Squid Quarterly Prog. Rep., U.S. Navy and U.S.A.F., July 1950.

6. Putnam, Abbott, A., and Denis, William R.: A Study of Burner Oscillations of the Organ-Pipe Type. Presented at Semiannual meeting A.S.M.E. (Cincinnati), June 15-19, 1952 .

7. Morse, Philip M.: Vibration and Sound. McGraw-Hill Book Co., Inc., 1936 .

8. Hunt, Frederick V.: Investigation of Room Acoustics by Steady-State Transmission Measurements. Jour. Acoustical Soc. Am., vol. 10, no. 3, Jan. 1939, pp. 216-227.

9. Merchaint, W.: Flow of an Ideal Fluid Past a Cascade of Blades. R.\&.M. No. 1890, British A.R.C., July 1940 .

10. Harris, Cyril N.: Acoustic Impedance Measurements of Very Porous Screens. Jour Acoustical Soc. An., vol. 20, no. 4, July 1948, pp. $440-447$. 
11. Dugger, Gordon I., and Heimel, Sheldon: Flame Speeds of Methane-Air, Propane-Air, and Ethylene-Air Mixtures at Low Initial Temperatures. NACA TN 2624, 1952 .

12. Gerstein, Melvin, Levine, Oscar, and Wong, Eagar L.: Fundamental Flame Velocities of Pure Hydrocarbons. I - Alkanes, Alkenes, Alkynes, Benzene, and Cyclohexane. MACA RM E50G24, 1950.

13. Karlovitz, Béla, Denniston, D. W., Jr., and Wells, F. E.: Investigation of Turbulent Flames. Jour. Chem. Phys. vol. 19, no. 5, May 195I, pp. 541-547.

14. Markstein, G. H.: Interaction of Flow Pulsations and Flame Propagation. Jour. Aero. Sciences, vol. 18, no. 6, June 1951, pp. 428429 .

15. Manton, John, von Elbe, Guenther, and Lewis, Bernard: Nonisotropic Propagation of Combustion Waves in Explosive Gas Mixtures and the Development of Cellular Flames. Jour. Chem. Phys., vol. 20, no. I, Jan. 1952, pp. 153-157.

16. Scurlock, A. C.: Flame Stabilization and Propagation in EighVelocity Gas Streams. MIT Meteor Report 19, Mass. Inst. Tech., May 1948. (Contract Nord 966I.) 


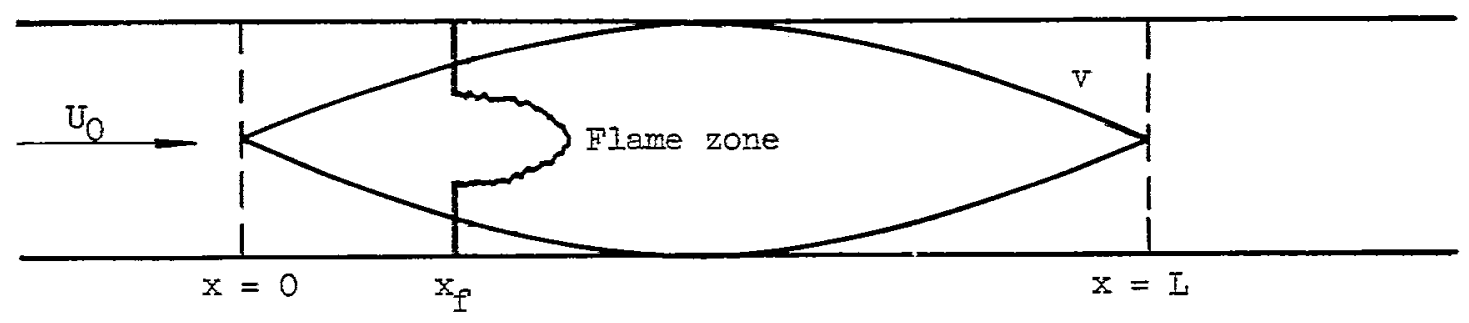

Figure 1. - Segment of duct between two pressure loops.

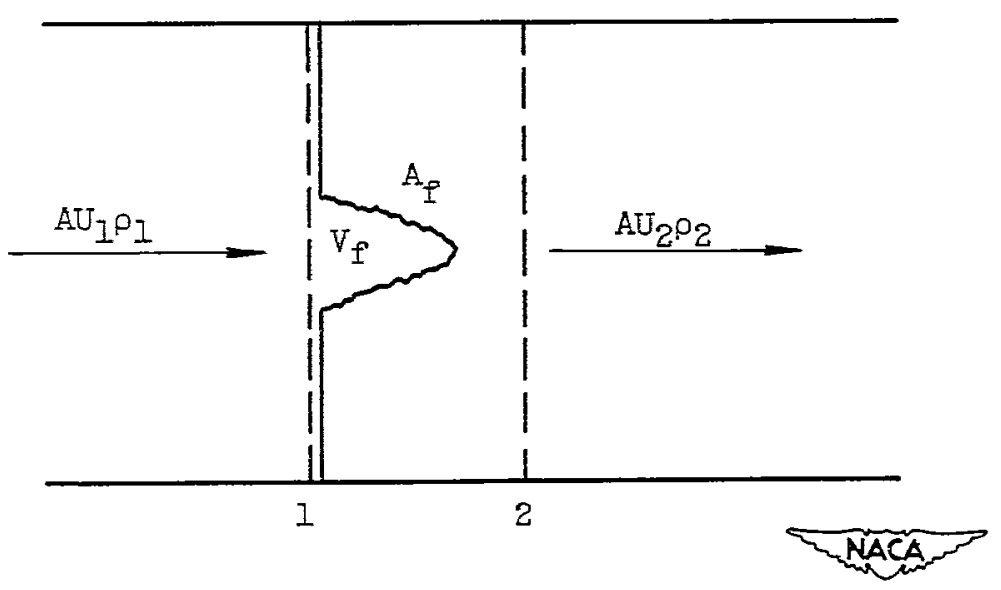

Figure 2. - Flame zone enlarged. 


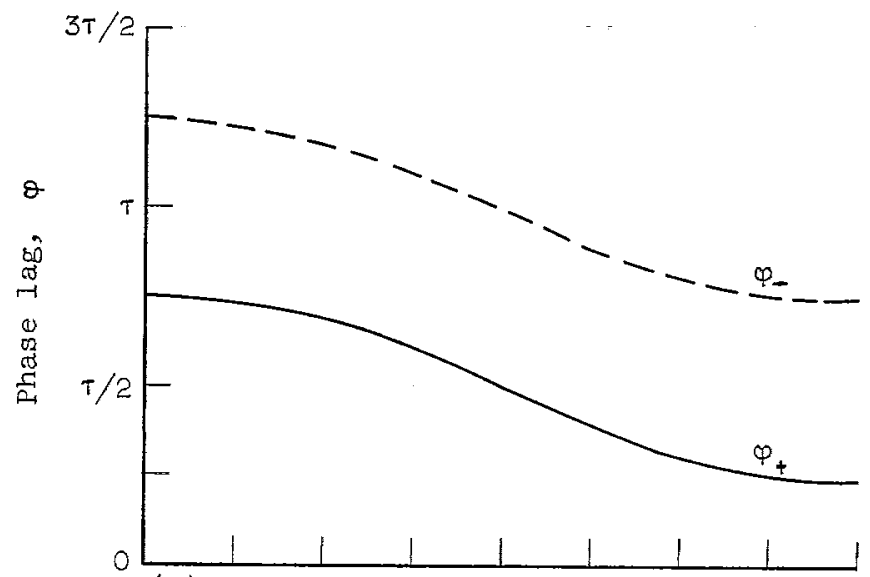

(a) Phase lag where maximum driving or damping occurs; $\frac{1-2 M_{0}}{\sqrt{T_{1} / T_{2}}+2 M_{0}}=\frac{1}{2}$.

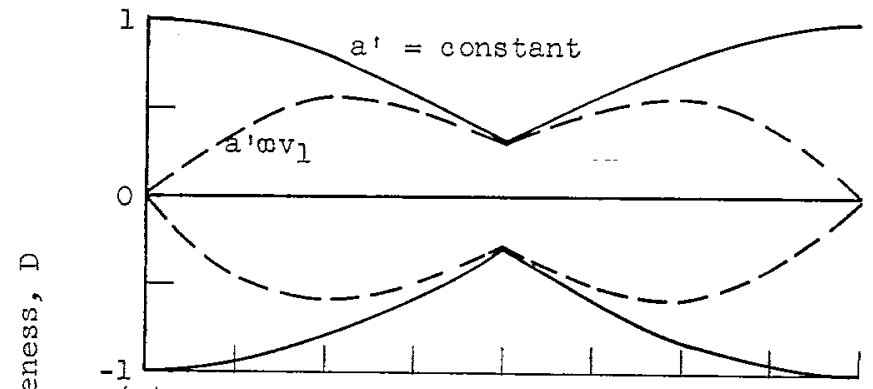

(b) Envelopes of driving effectiveness for $a^{\prime} \omega v_{1}$ and $a^{\prime}=$ constant;

$$
\frac{1-2 M_{0}}{\sqrt{T_{1} / T_{2}}+2 M_{0}}=\frac{1}{2} \text {. }
$$

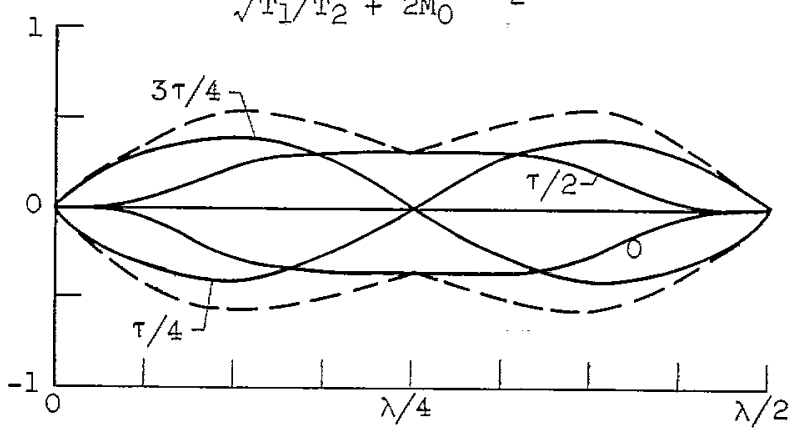

Flame-holder position measured from pressure loop, $\mathrm{x}_{\mathrm{f}}$

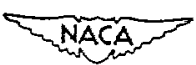

(c) Driving effectiveness for representative values of $\varphi ; \frac{1-2 M_{O}}{\sqrt{T_{1} / T_{2}}+2 M_{0}}=\frac{1}{2}$. (Numbers beside curves are values of $\varphi$. )

Figure 3. - Phase-lag requirements for maximum effectiveness. 
ลูง
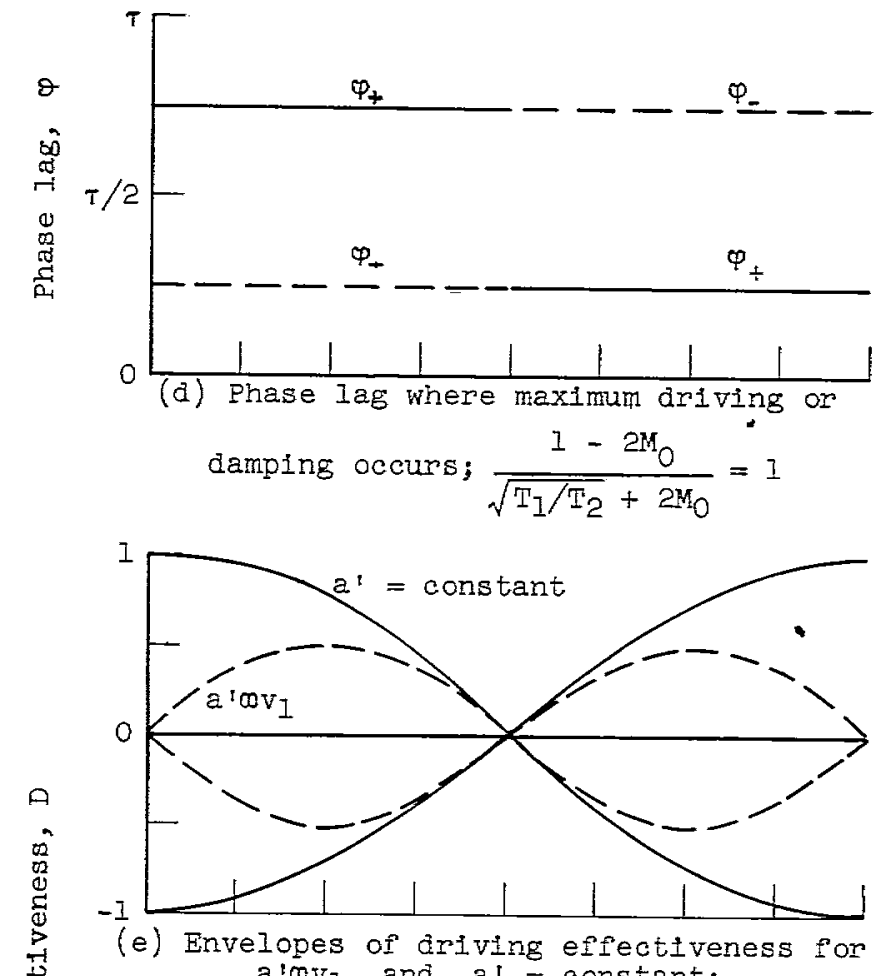
$a^{\prime} \infty v_{1}$ and $a^{\prime}=$ constant;

$$
\frac{1-2 M_{0}}{\sqrt{T_{1} / T_{2}}+2 M_{0}}=I
$$

$\stackrel{\infty}{\stackrel{8}{3}}$

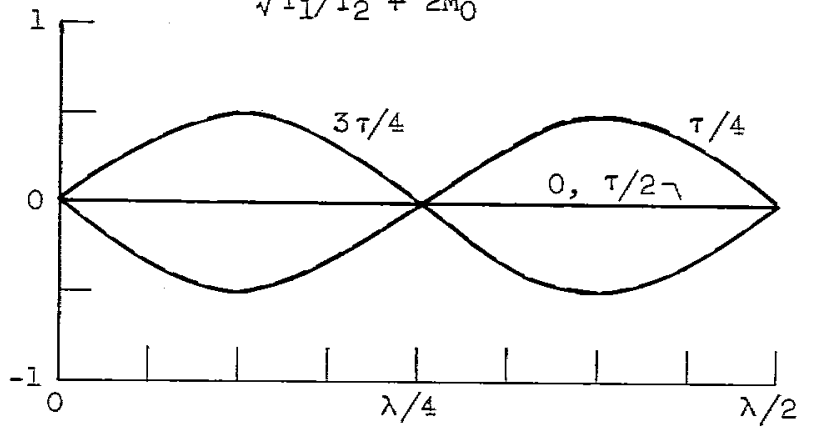

Flame-holder position measured from pressure loop, $\mathrm{x}_{\hat{\mathrm{I}}}$

(f) Driving effectiveness Ior representative values of $\varphi ; \frac{1-2 M_{O}}{\sqrt{T_{1} / T_{2}}+2 M_{O}}=1$. (Numbers
besice curves are values of $\varphi$.) NACA Figure 3. - Continued. Phase-lag requirements for maximum effectiveness. 


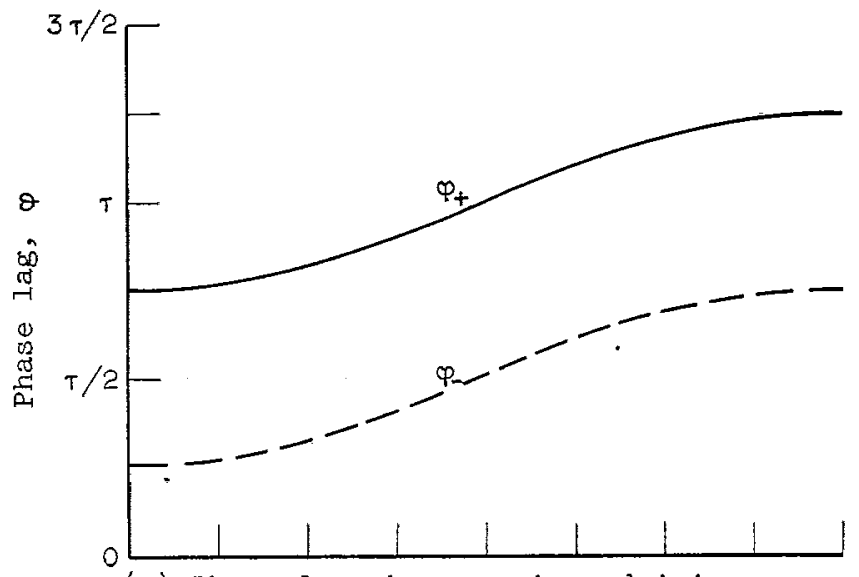

(8) Phase lag where maximum driving or damping occurs; $\frac{1-2 M_{O}}{\sqrt{T_{1} / T_{2}}+2 M_{O}}=2$.

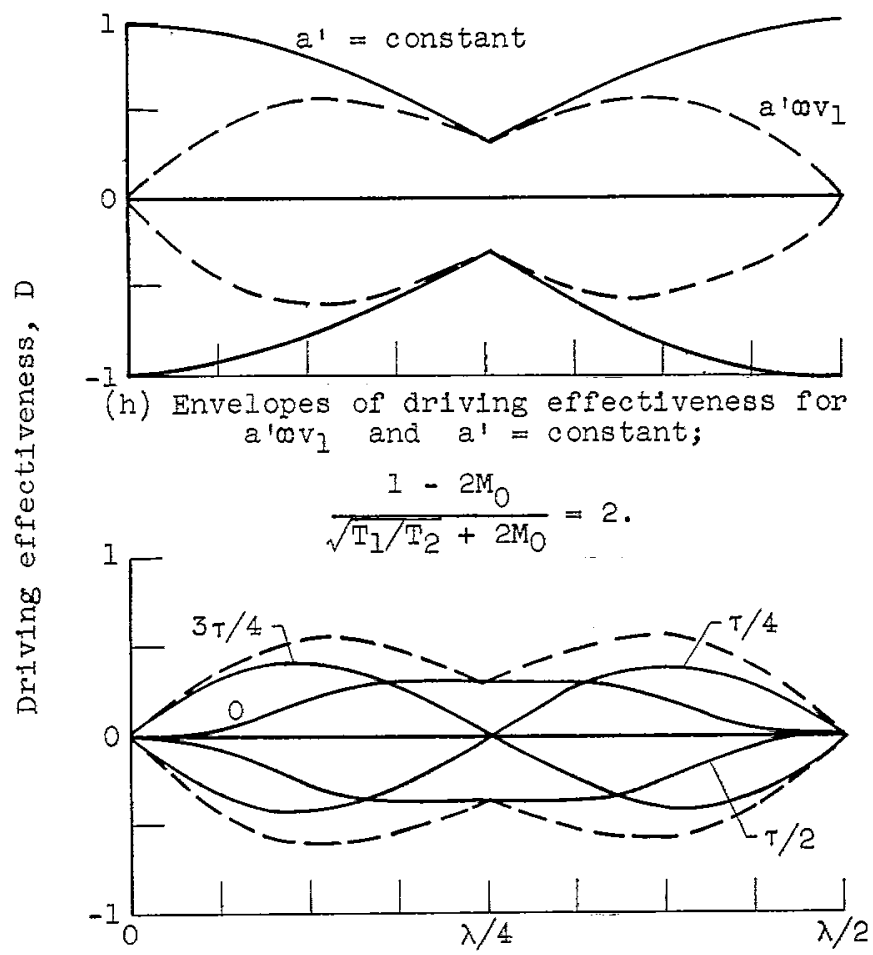

Flame-holder position measured from pressure $100 p, x_{f}$

(1) Lriving effectiveness for representative values of $\varphi ; \frac{1-2 M_{0}}{\sqrt{T_{1} / T_{2}}+2 M_{0}} \equiv 2$. (Numbers beside curves are values of $\varphi_{\text {.) }}$ NACAN

F1gure 3. - Concluded. Phase-lag requirements for maximum effectiveness. 

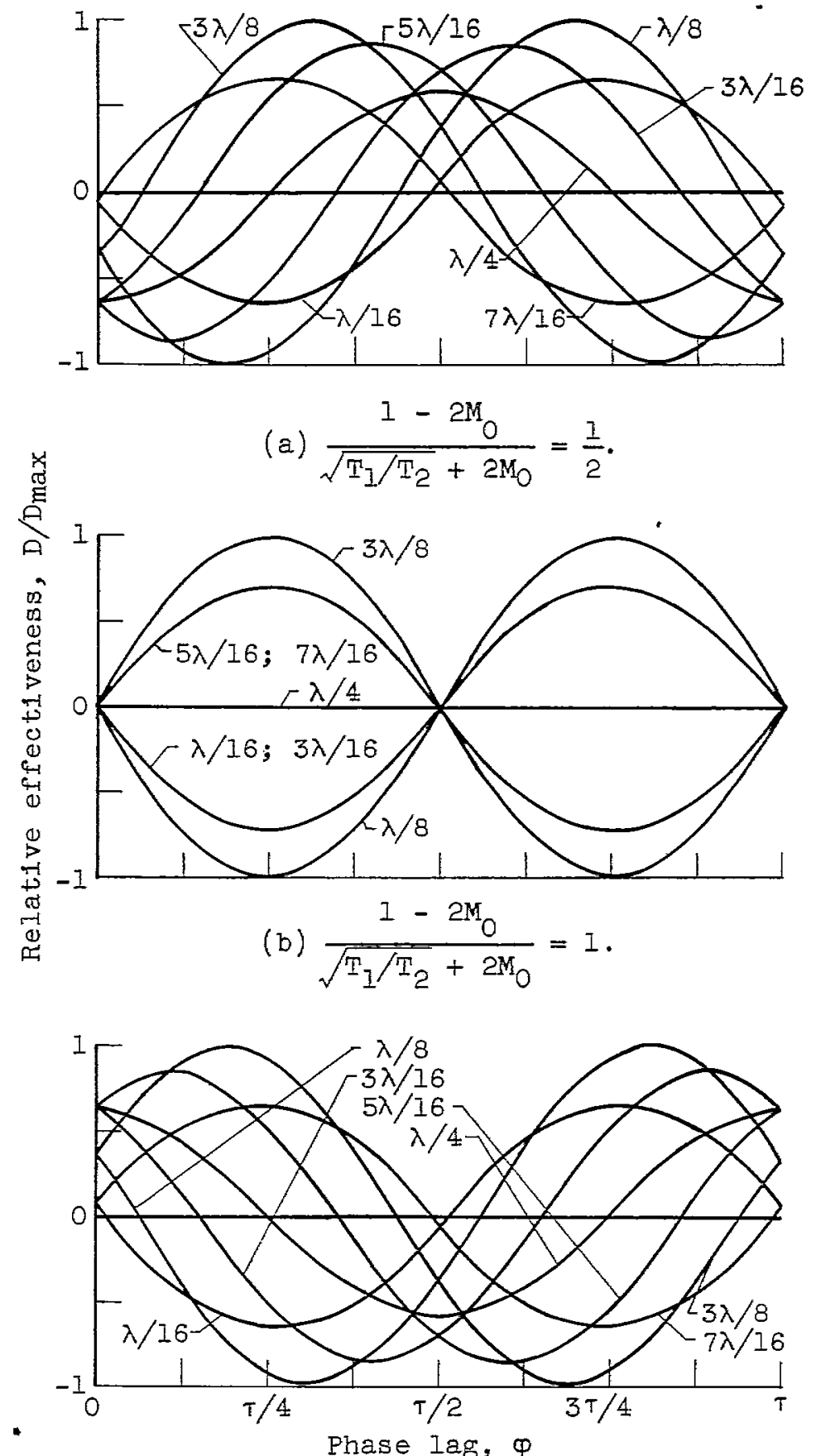

(c) $\frac{1-2 M_{0}}{\sqrt{T_{1} / T_{2}}+2 M_{0}}=2$. UNACA-

Figure 4. - Relative effectiveness at given $x_{f}$ for variations in $\varphi$. (Numbers beside curves are values of $x_{f} \cdot$ ) 


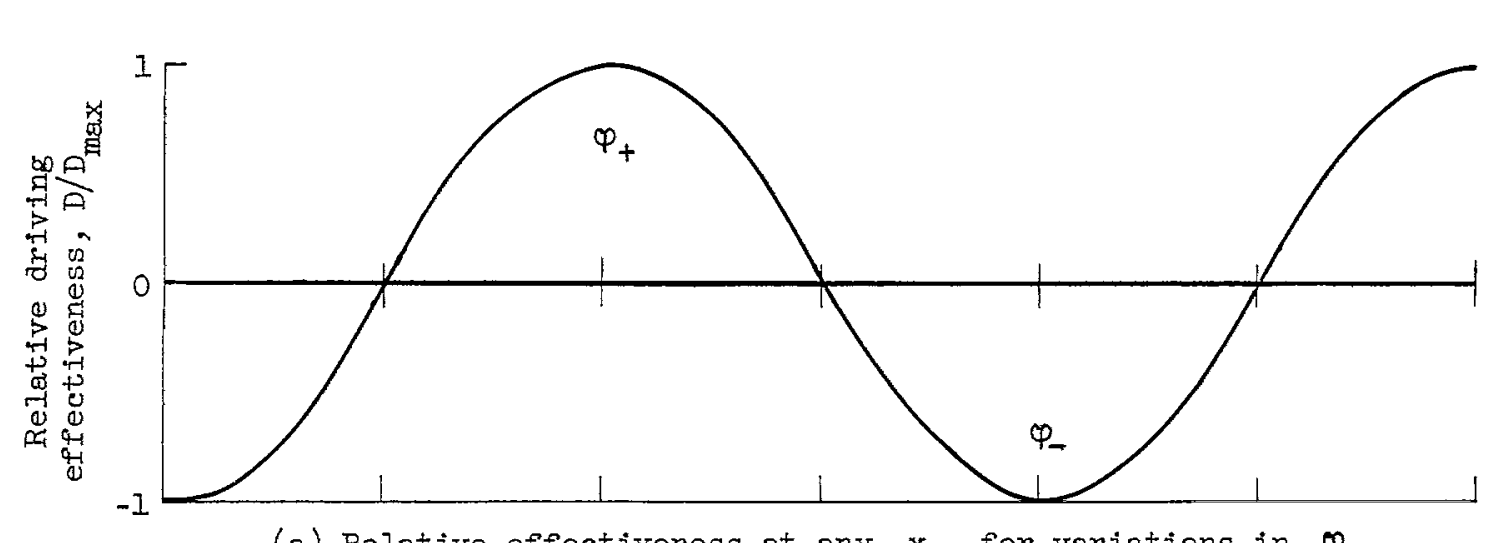

管

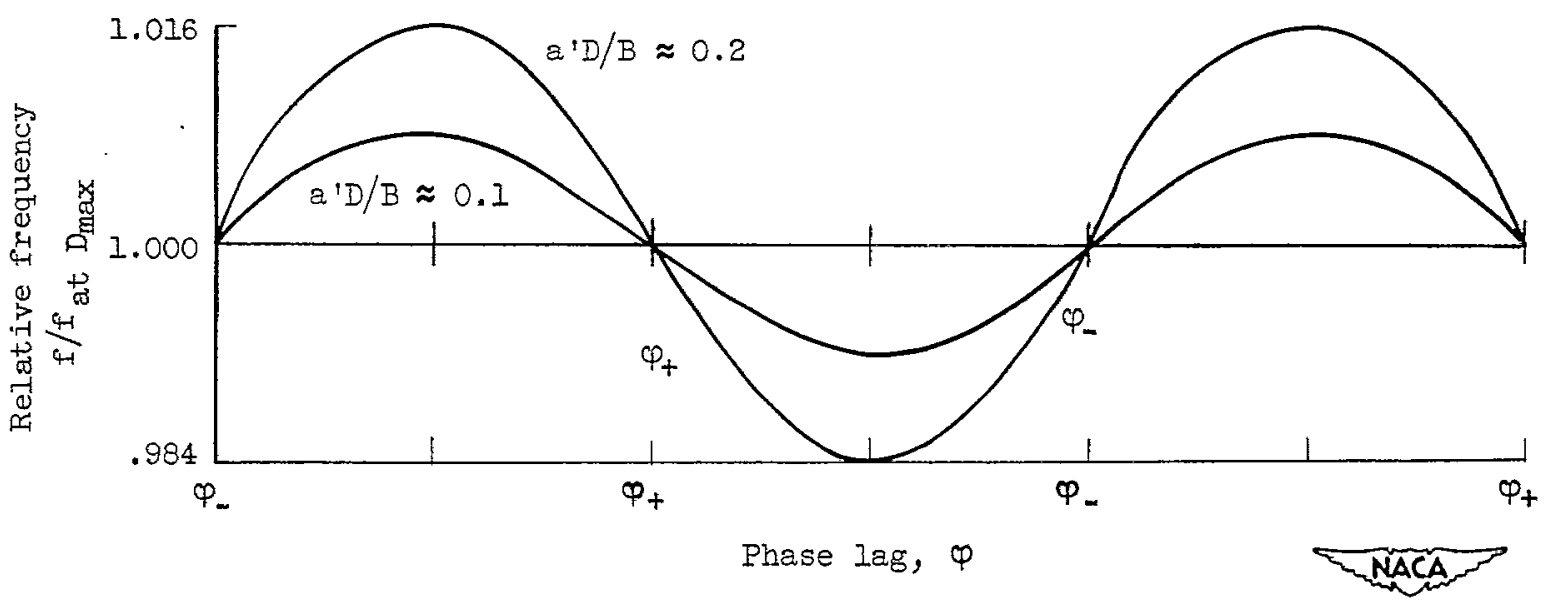

(b) Relative frequency at any $x_{f}$ for variations in $\phi$.

Figure 5. - Relative driving effectiveness and frequency for any $x_{f}$ against $\varphi$ relative to $\varphi_{+}$for the $x_{f}$ chosen. 


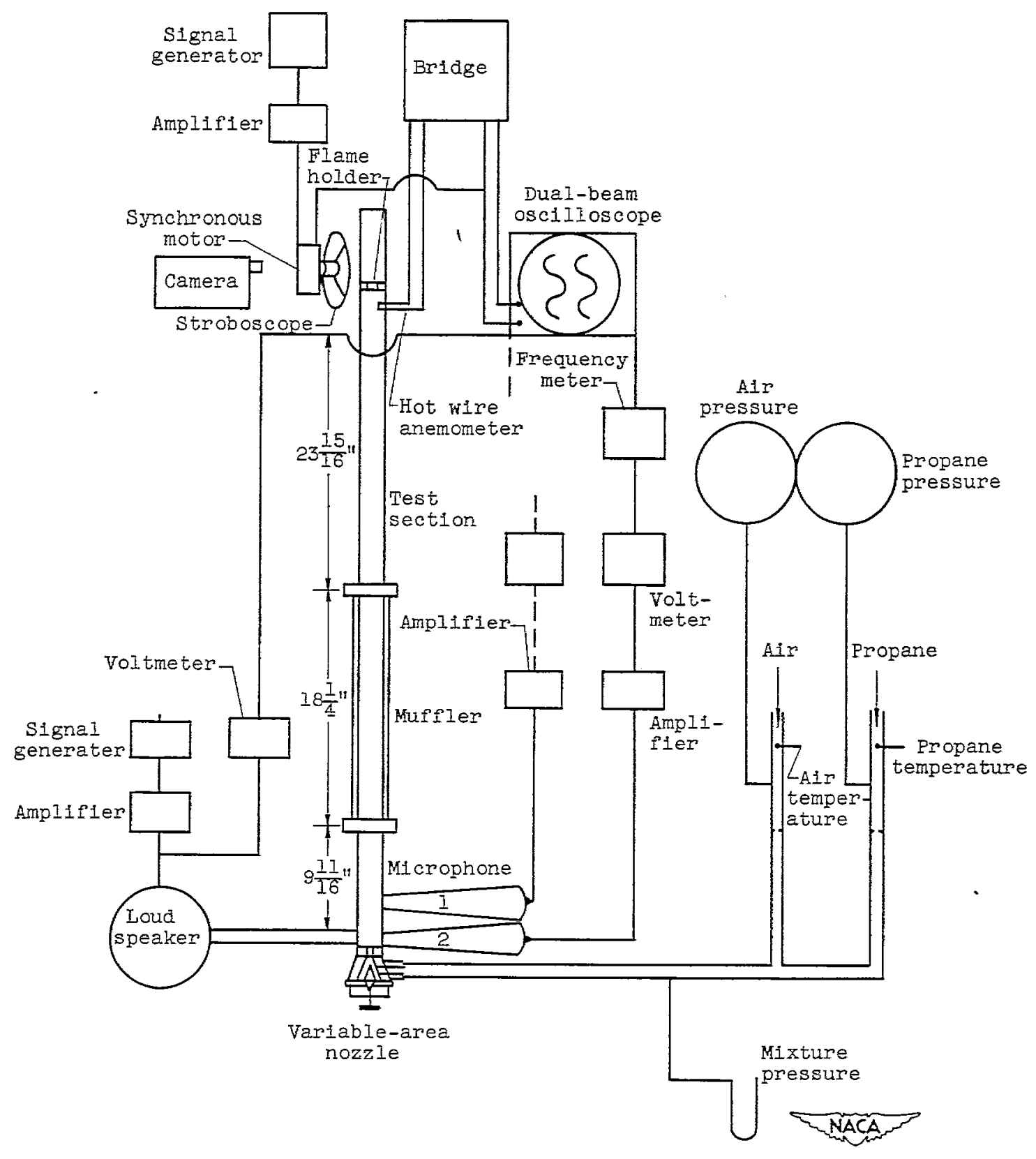

Flgure 6. - Test apparatus. 

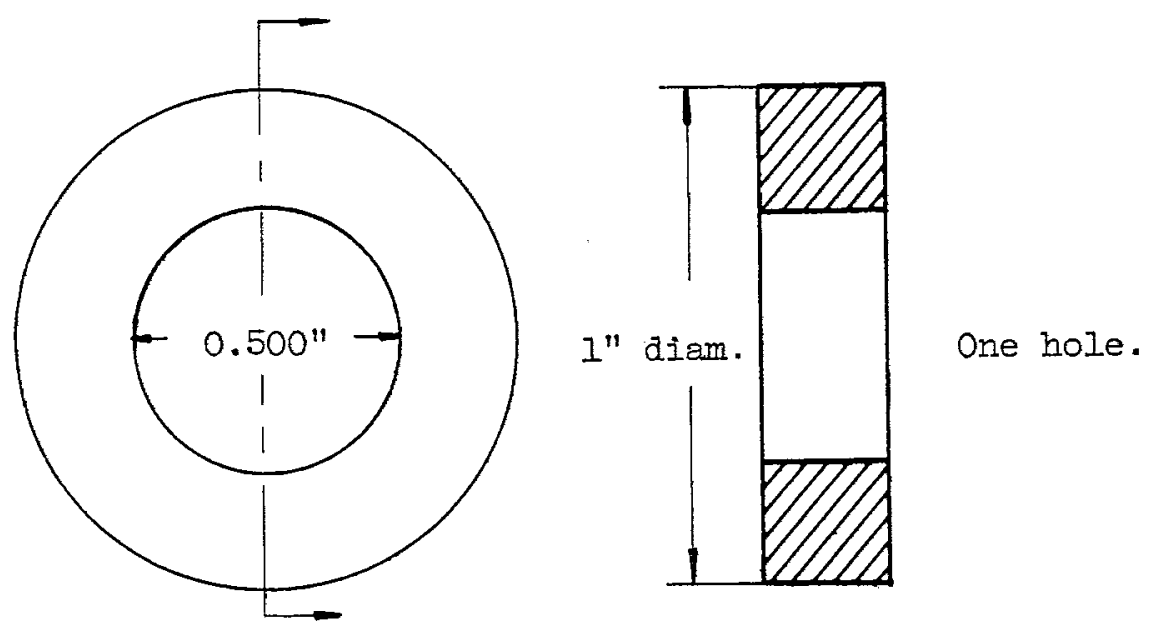

$N$
0
0
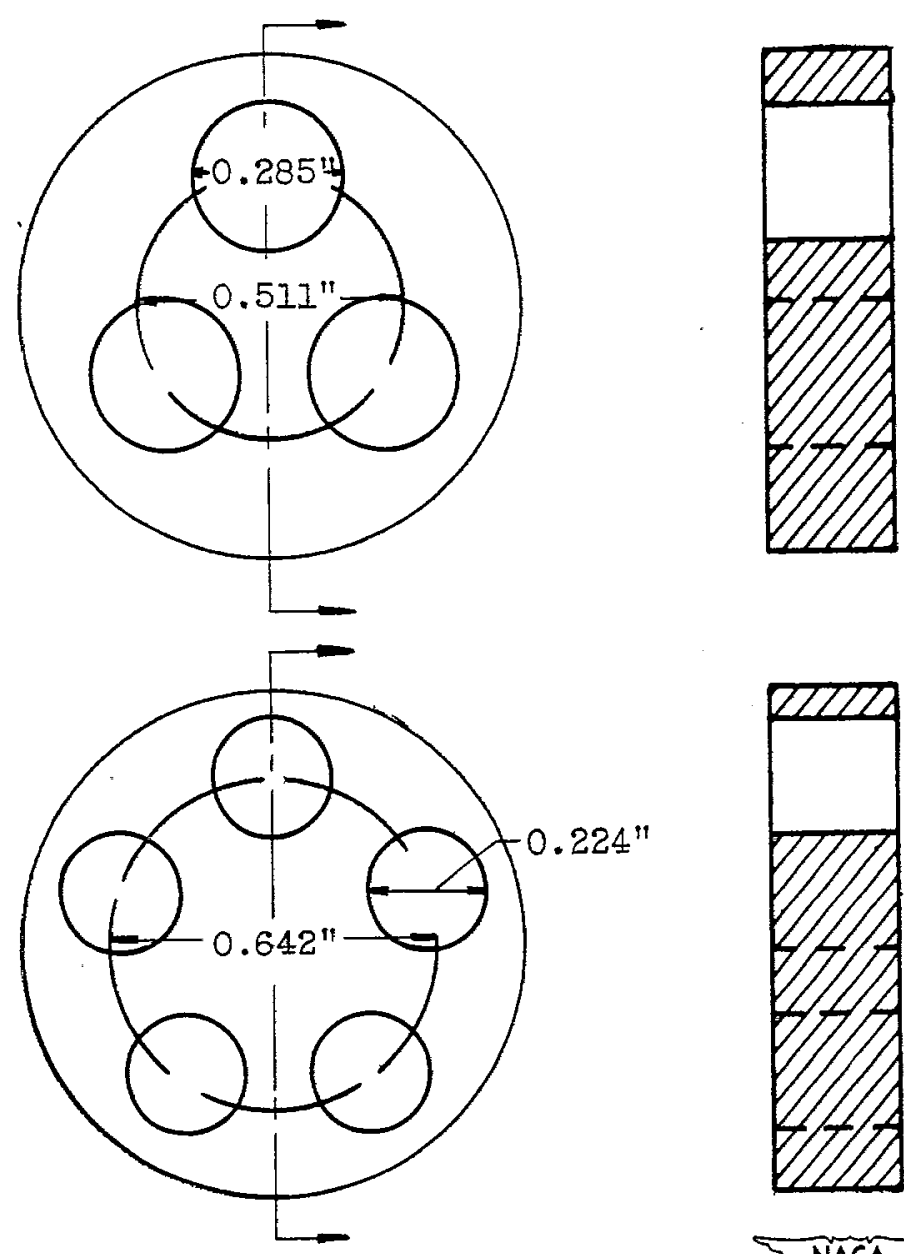

Three hole.

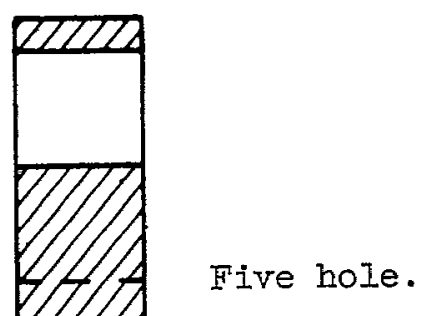

Figure 7. - Flame holders. 


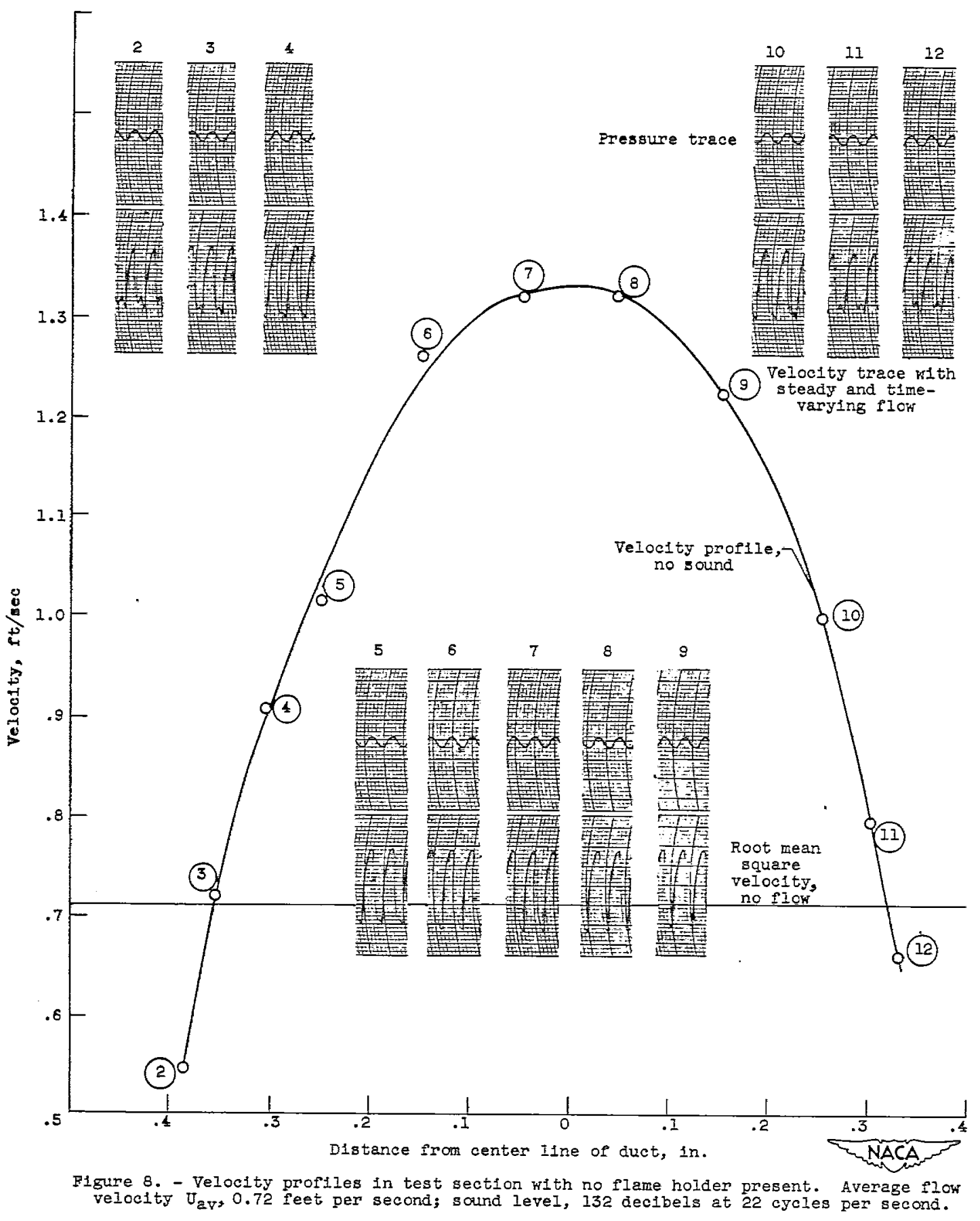




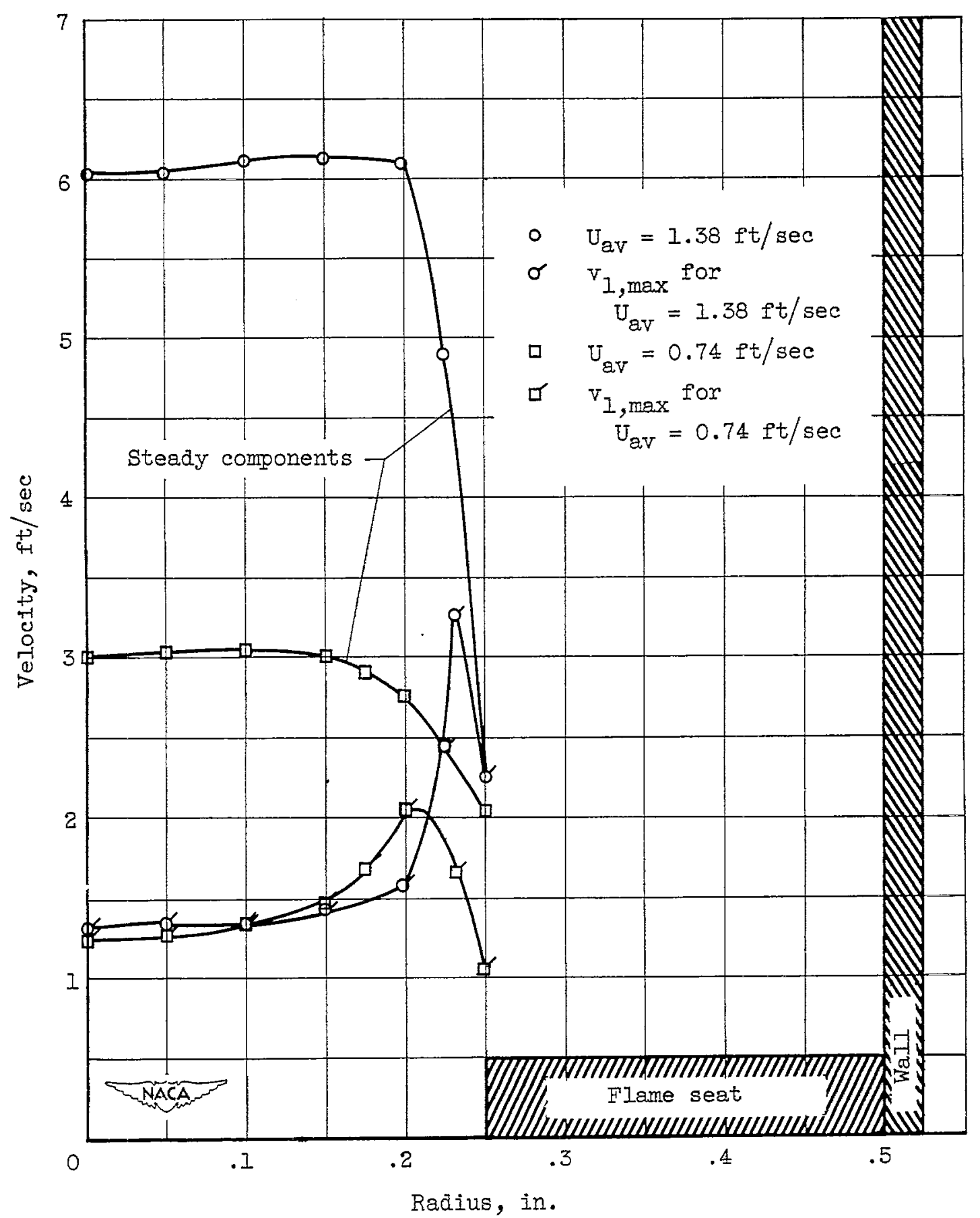

Figure 9. - Velocity profiles measured 1/16 inch downstream of onehole flame holder placed 4 inches from exhaust at 55 cycles per secona. 


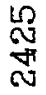

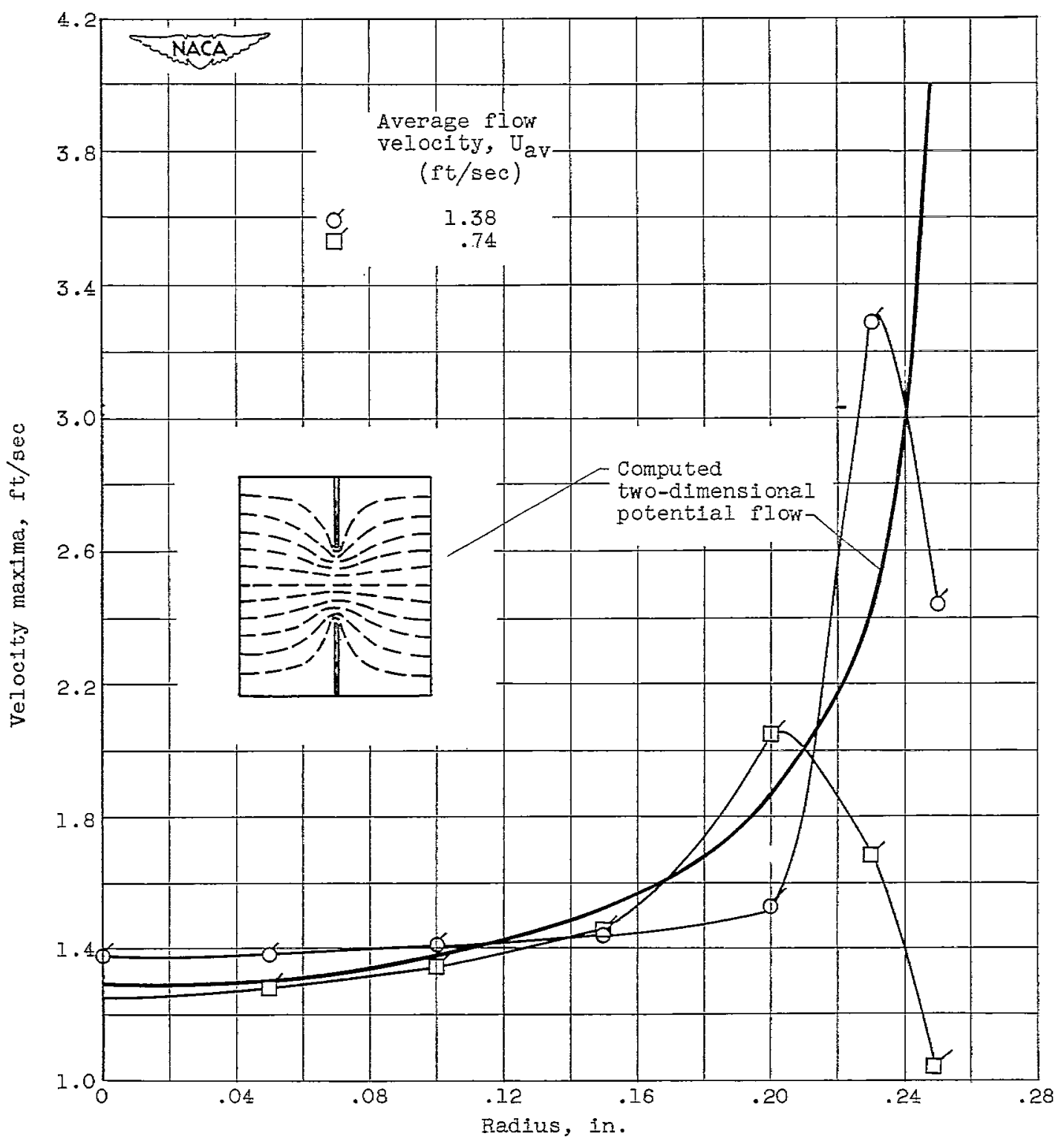

Figure 10. - Comparison of measured time-varying flow through flame holder (see fig. 9) with two-dimensional potential flow. 


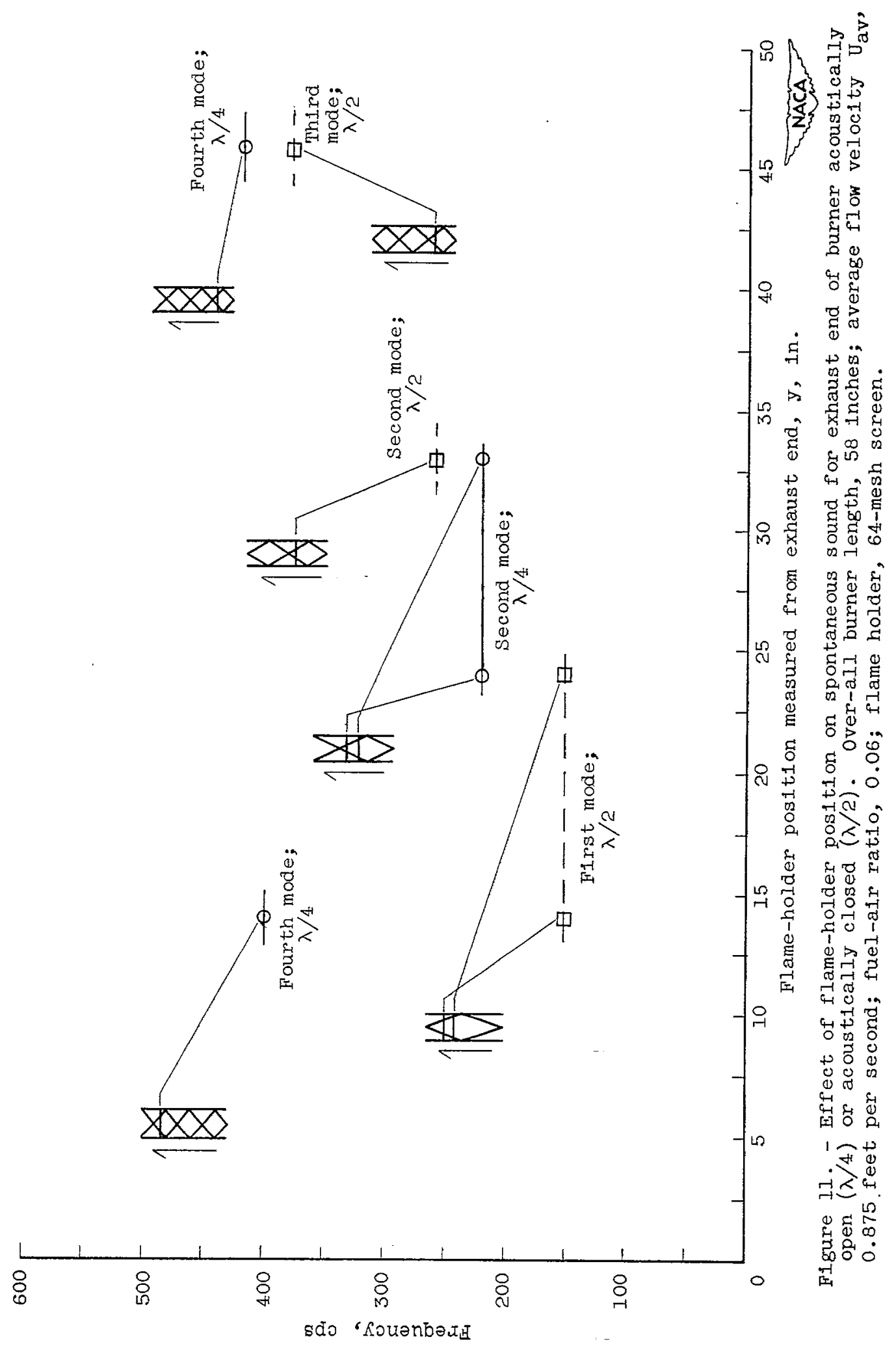




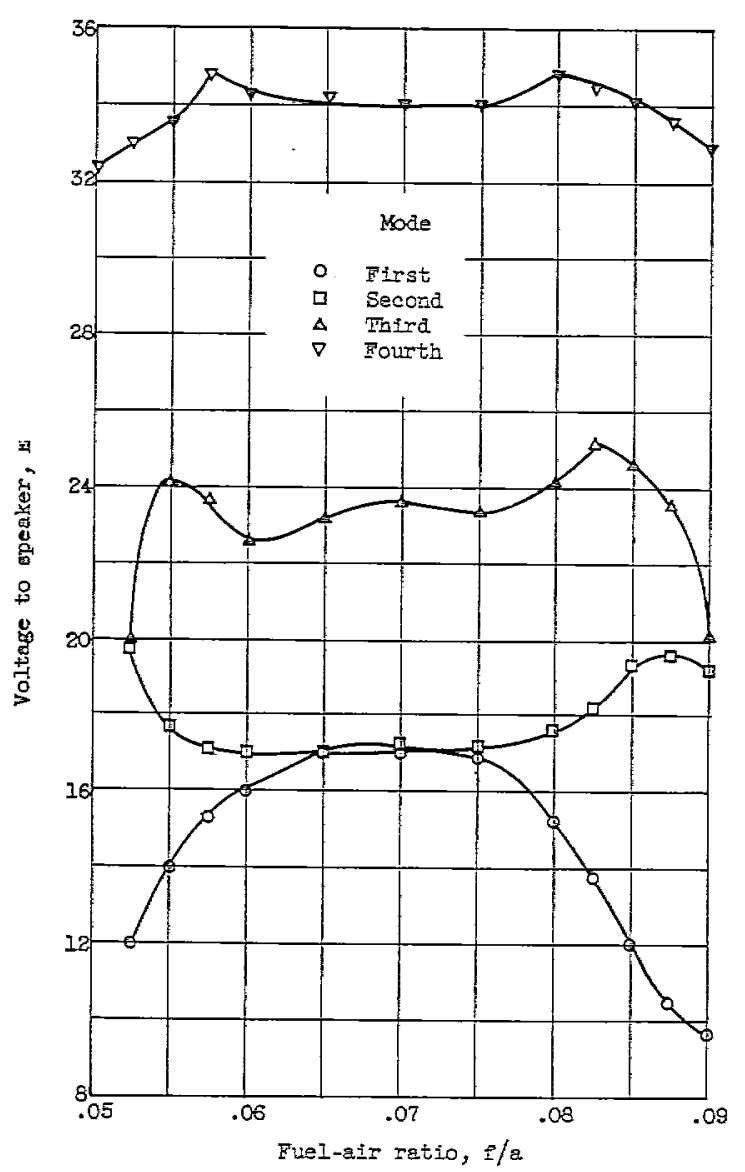

(e) One-hole flame holder.

Figure 12. - Voltage to sieeker recuired to arive Durner at 123.2-decibel sound level at resonance for Pirst four modes of vibretion. Flame-holcer position measured from exhsust ena $\mathrm{y}, \leqslant$ inches; average $\mathrm{PI}$ (Wr velocity $\mathrm{J}_{\mathrm{ev}}, 1.51$ jeet per second.

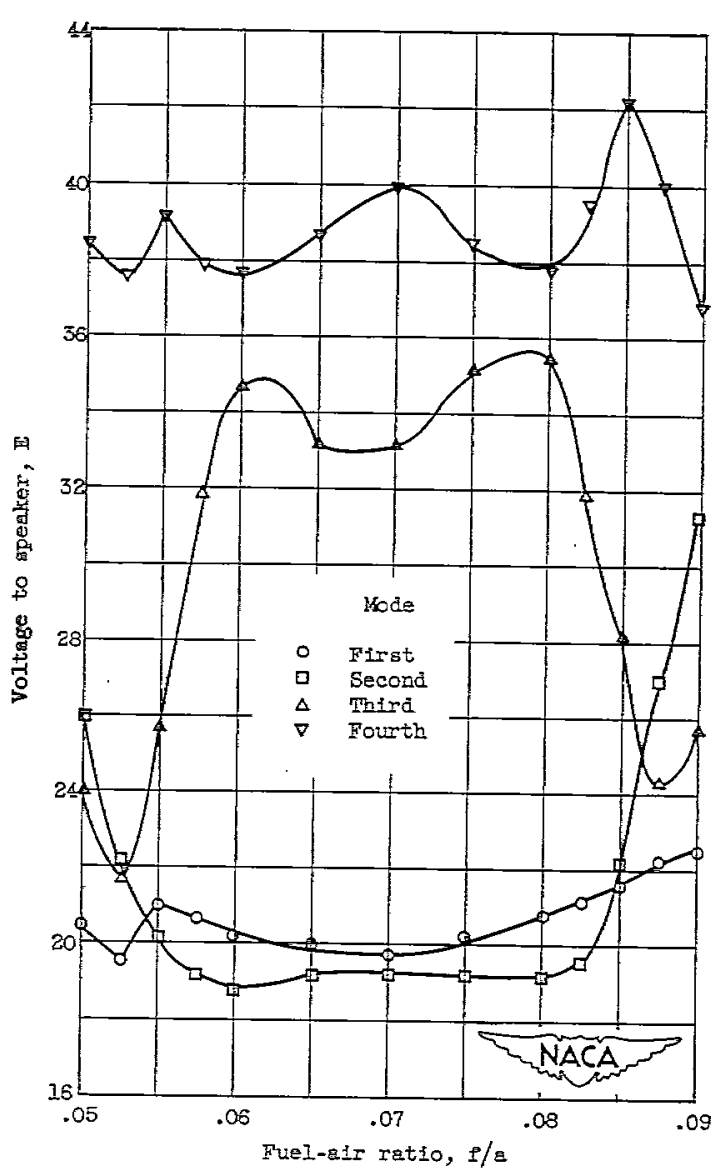

(b) Three-hole flame holder.

Figure 12. - Continued. Voltage to speaker required to drive burner at 123.2 -decibel sound bration. Flane-holder position measured from exhaust end $y, 4$ inches; averege flow relocity $\mathrm{U}_{\mathrm{av}}, 1.51$ leet per second. 


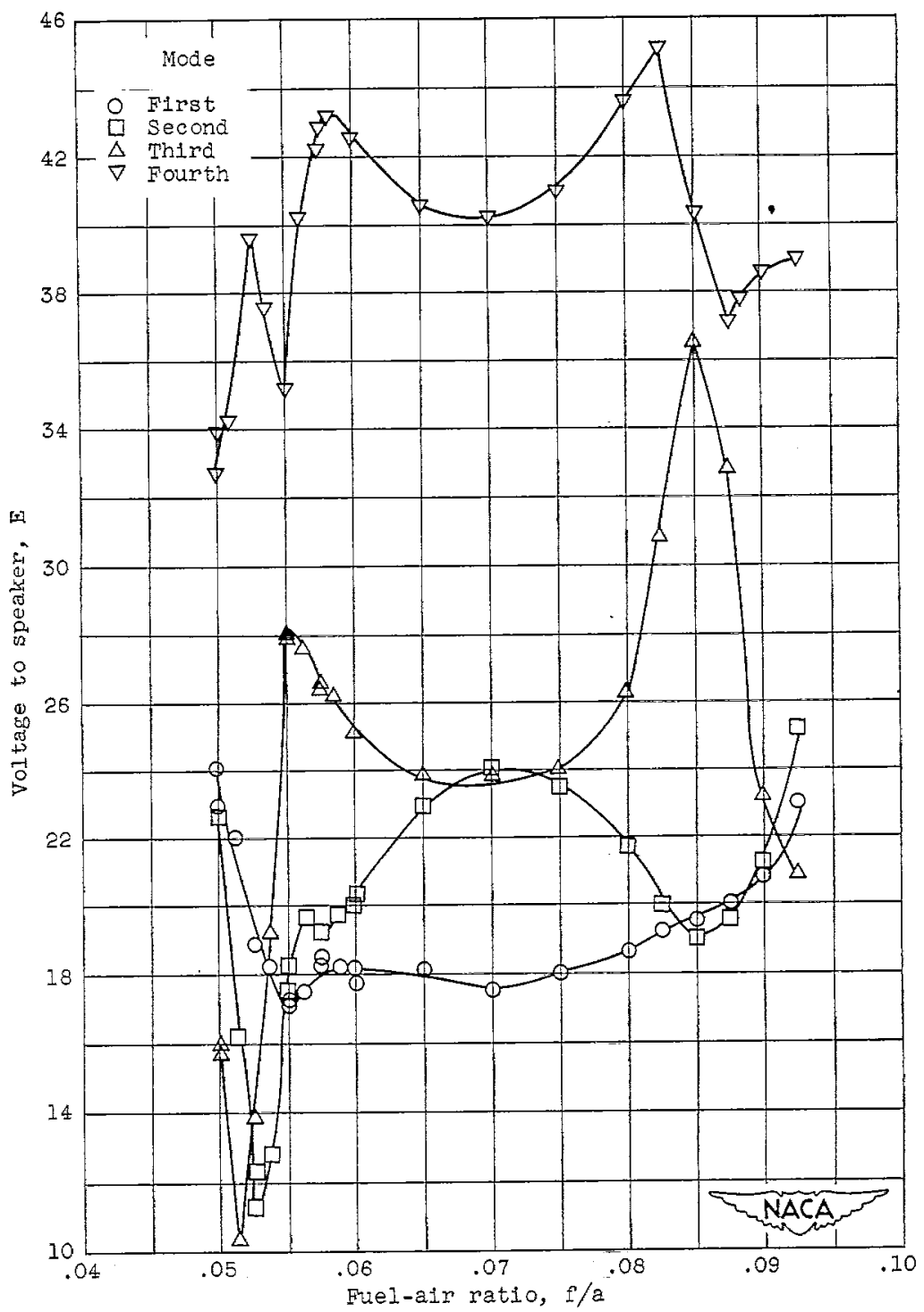

经

(c) Five-hole flame holder.

Figure 12. - Continued, Voltage to speaker required to drive burner at 123.2-declbel sound level at resonance for first

four modes of vibration. Flame-holder position measured

from exhaust end $y, 4$ inches; average flame velocity $U_{a y}$,

1.51 feet per second. 

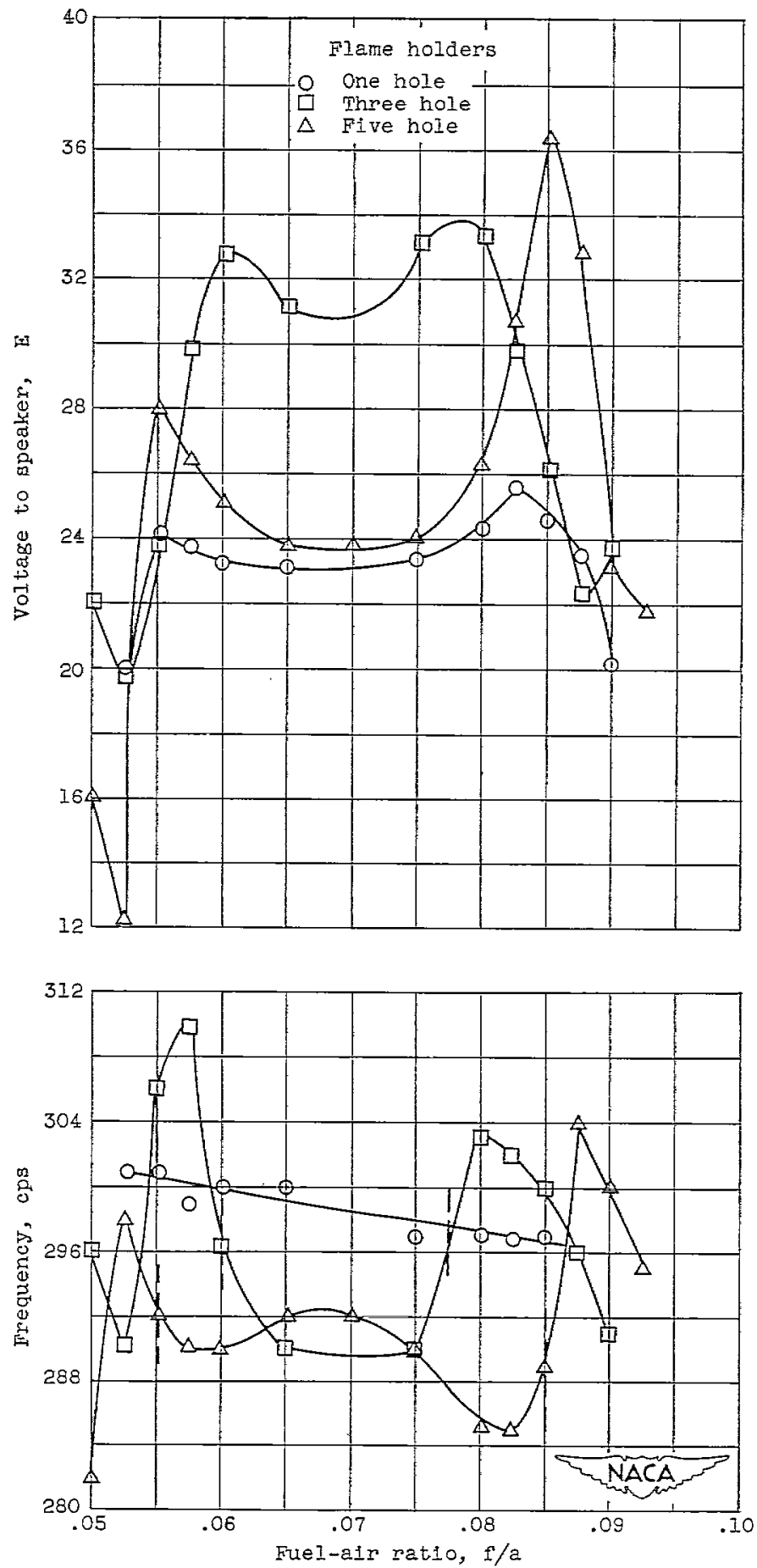

(d) Frequency shift compared with damping. Third mode.

Figure 12. - Concluded. Voltage to speaker required to drive burner at 123.2-decibel sound level at resonance for eirst Four modes of vibration. Flame-holder position measured from exhaust end $y$, 4 inches; average flow velocity $U_{a v}, 1.51$ feet per second. 


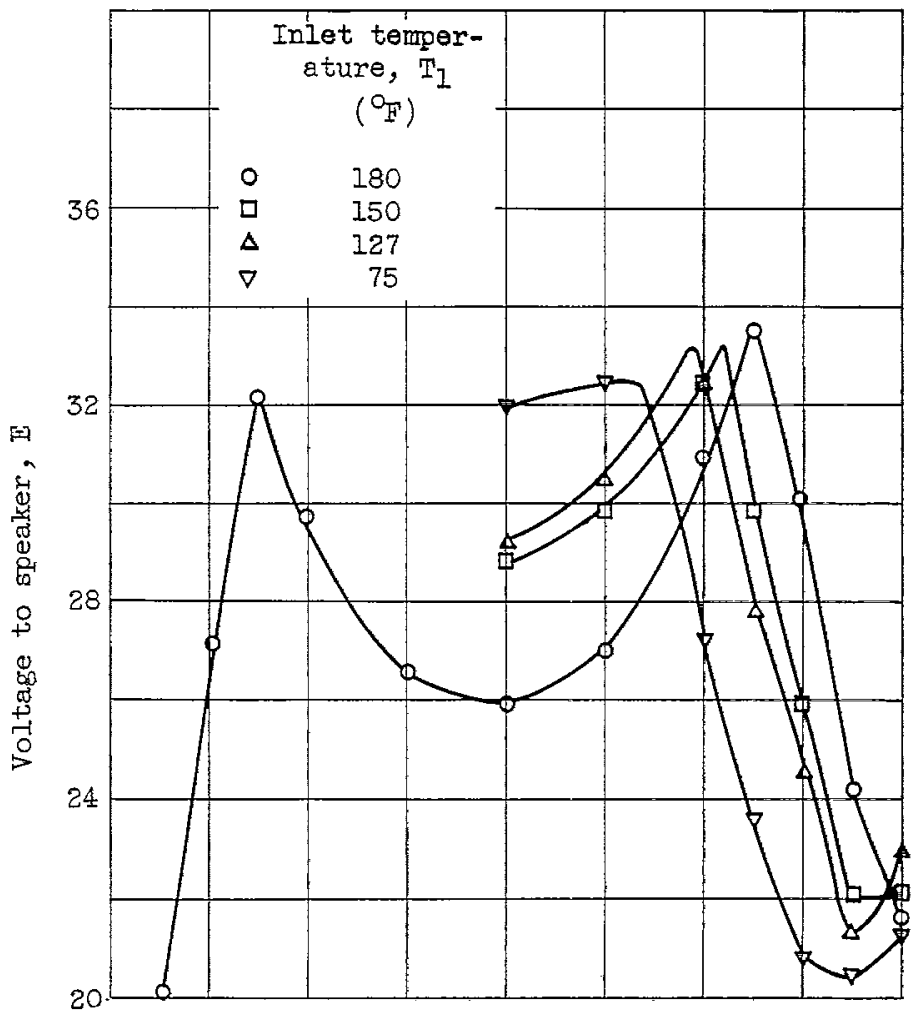

跑

(a) Thind mode.

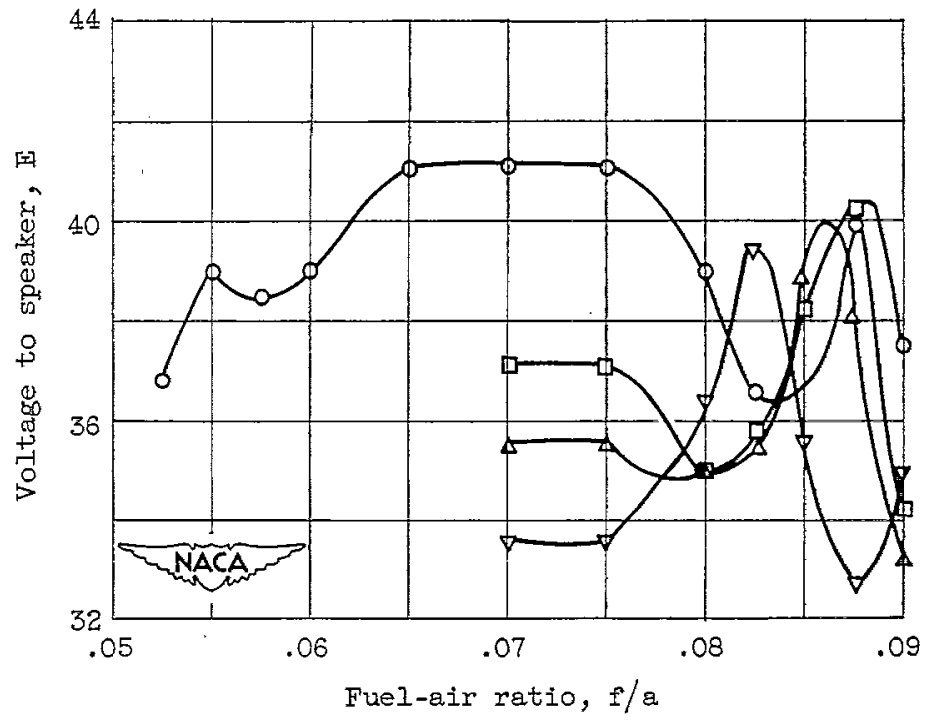

(b) Fourth mode.

Figure 13. - Efiect of inlet temperature on damping for three-hole flame holder in glass tube. Flame-holder position measured from exhaust end $\mathrm{y}, 4$ inches; average flow velocity $U_{a v}$,

1.51 feet per second; sound level, 123 decibels. 


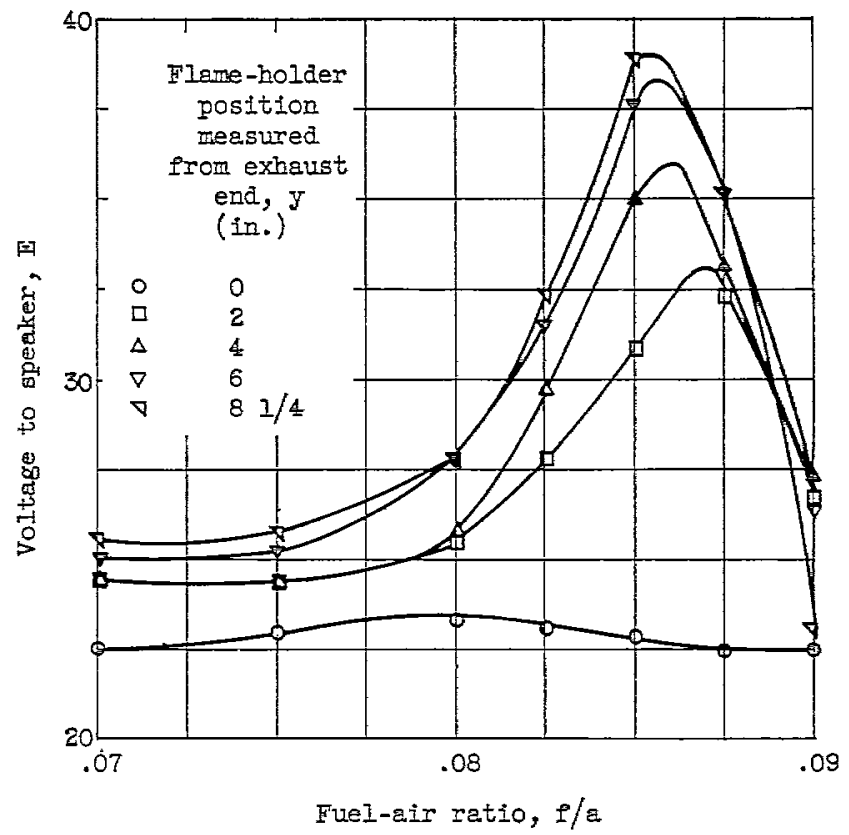

Figure 14. - Effect of flame-holder position on damping for three-hole flame holder. Third mode.

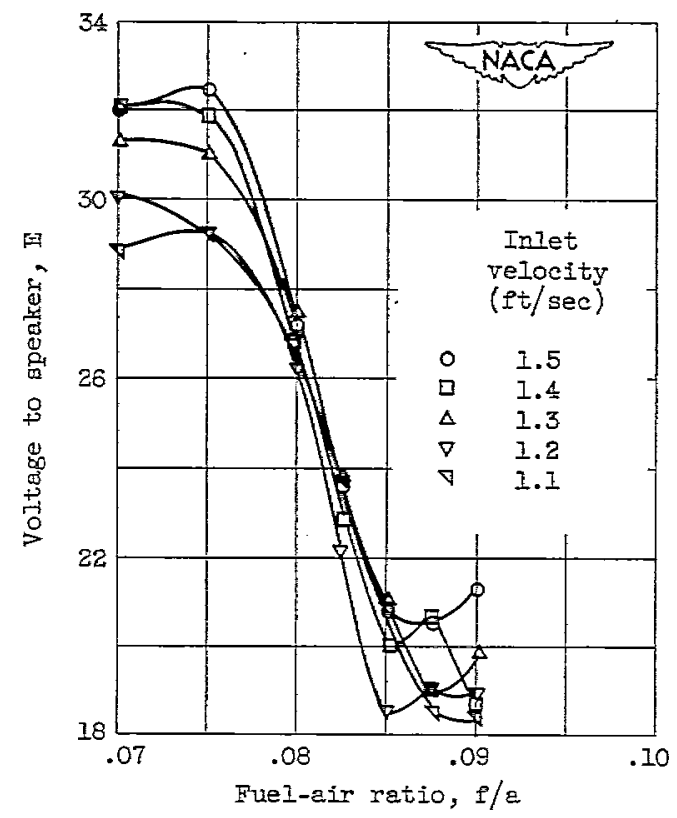

Figure 15. - Effect of inlet velocity on darroing for three-hole flame holder in glass tube. Third mode; flame-holder position measured from exhaust end $y, 4$ inches; sound level, I23 decibels. 

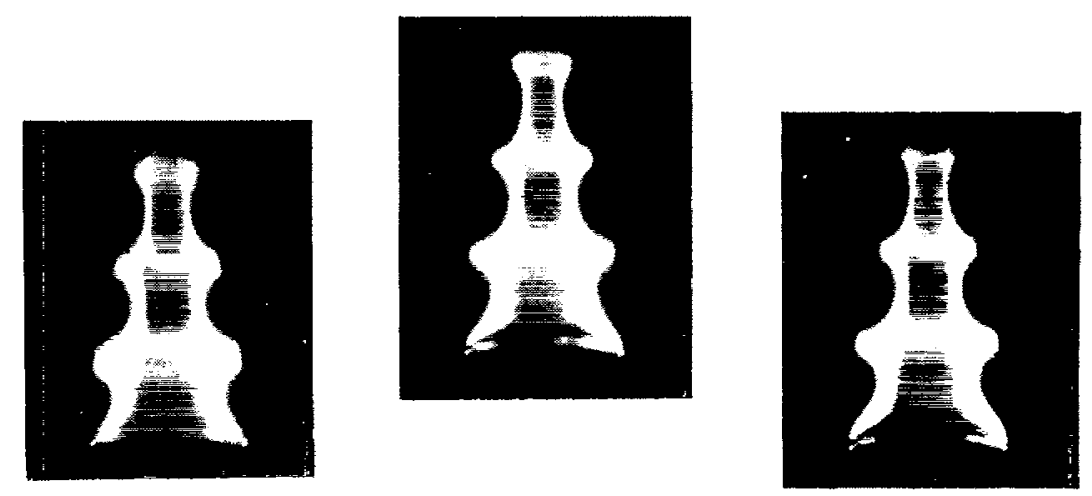

$\underset{N}{N}$
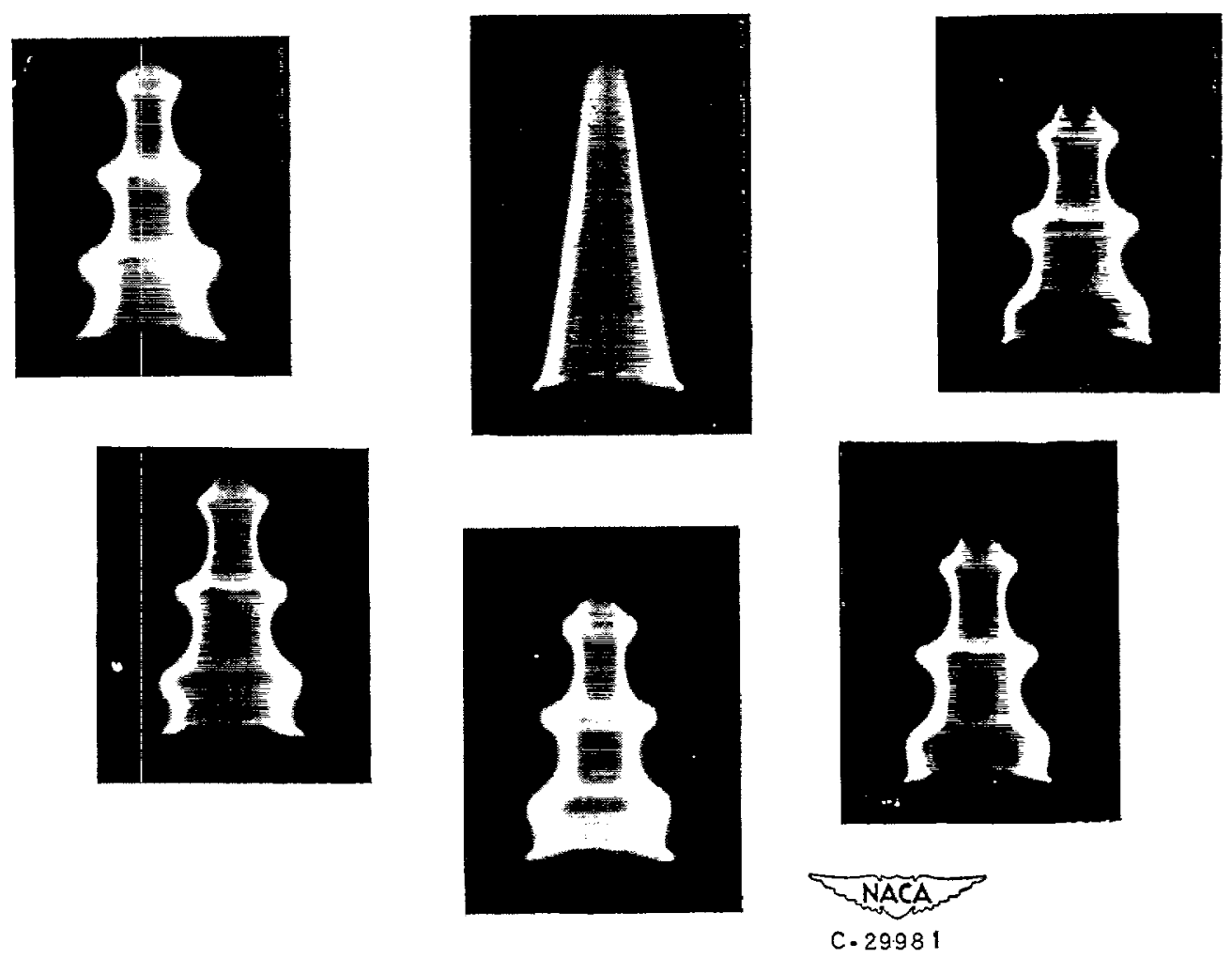

(a) Frequency, 329 cycles per second; voltage, 23.8 volts; velocity, 1.378 feet per second; fuel-air ratio; 0.080 .

Figure 16. - Sequence of flame shapes for one cycle at intervals of $45^{\circ}$. Time increases clockriae. 

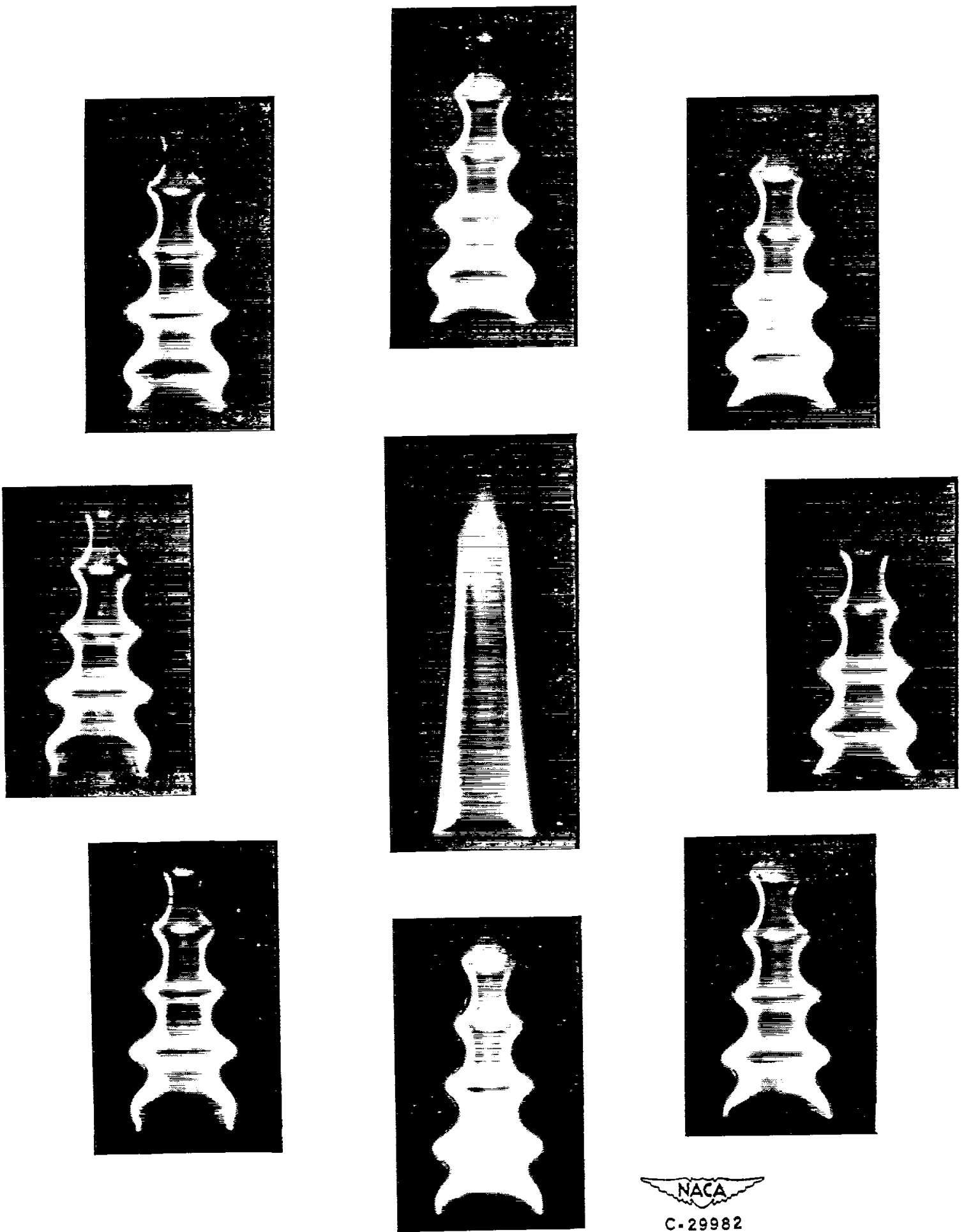

C. 29982

(b) Frequency, 311 cjoles per second; voltage, 25.3 volts; velocity, 1.378 feet per second; Iuel-air ratio, 0.090 .

Figre 16. - Continued. Sequence of flame sinapes for one cycle at intervals of $45^{\circ}$. Time increases clockwise. 

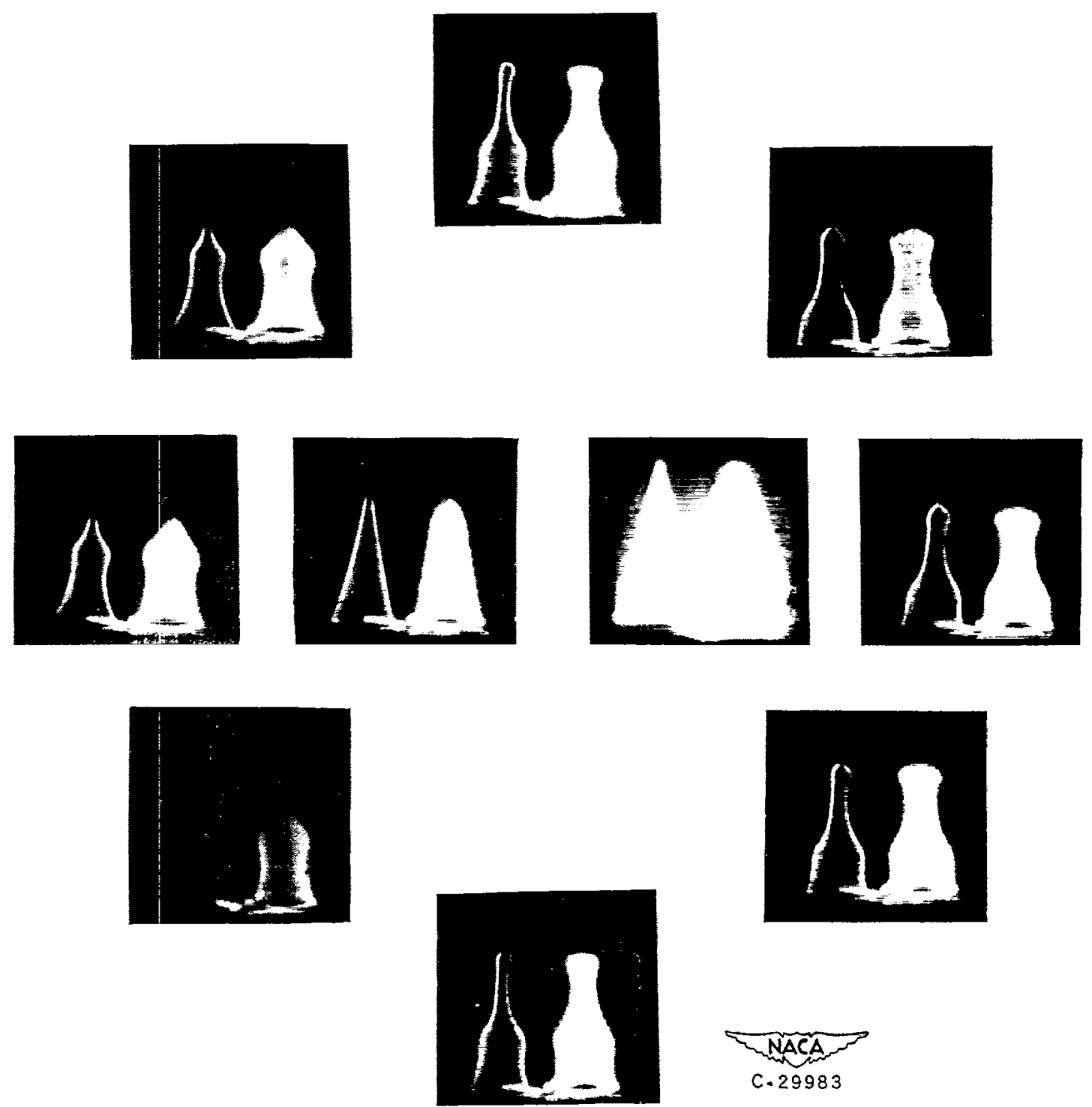

(o) Erequency, 318 cycles per second; voltage, 32.0 rolts; velocity, 1.378 feet per second; fuel-air ratio, 0.0775; run 2 (fig. 17).

Figure 16. - Continued. Sequence of flame shapes for one cycle at intervals of $45^{\circ}$. Time increases clockwise. 

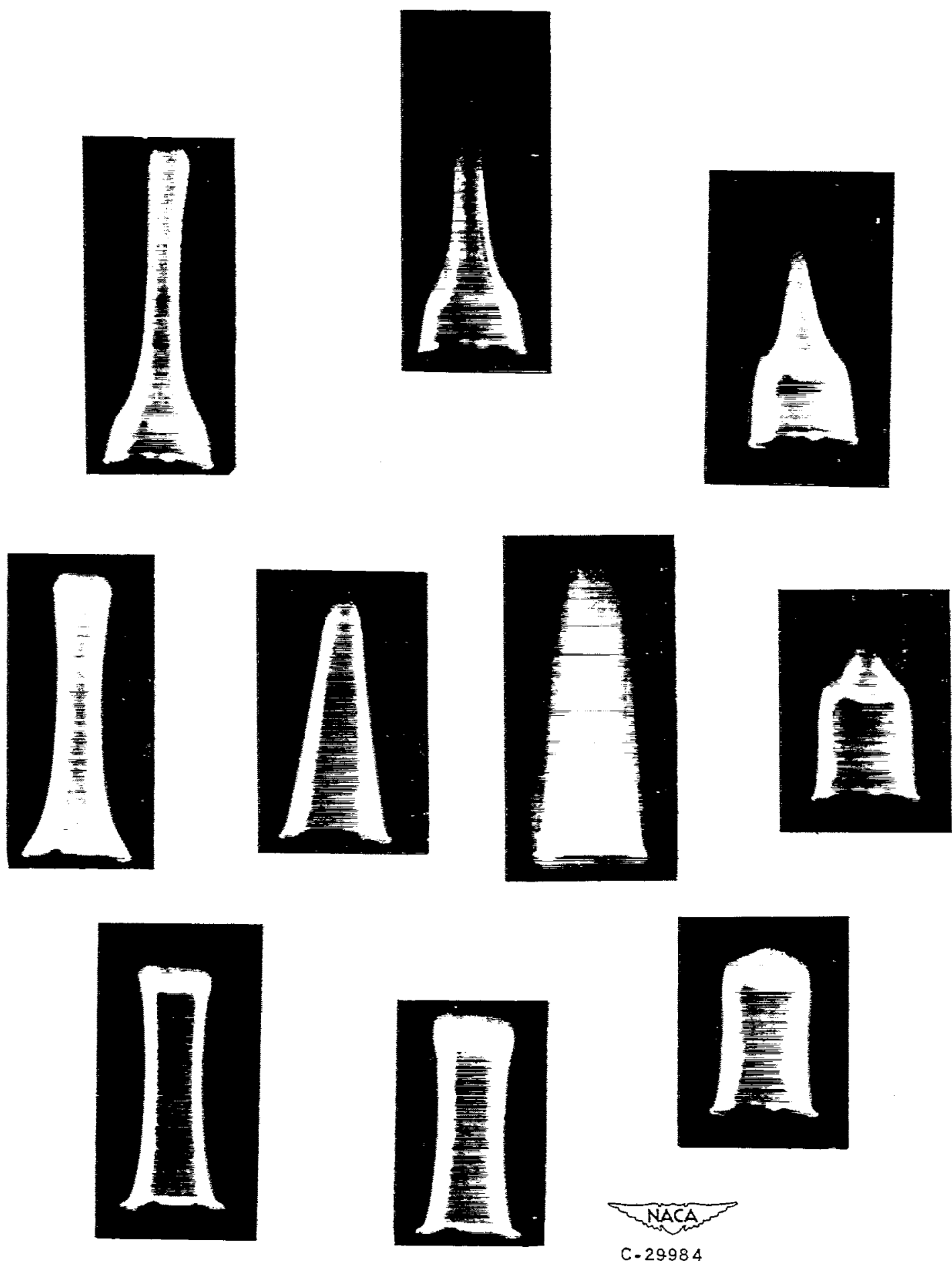

(d) Frequency, 58.5 cycles per second; voltage, 17.3 rolìs; velocity, 1.378 feet per second; fuel-air ratio, 0.080 .

Figure 16. - Continued. Sequence of flame shapes for one cycle at intervals of $45^{\circ}$. Time increases clockwise. 

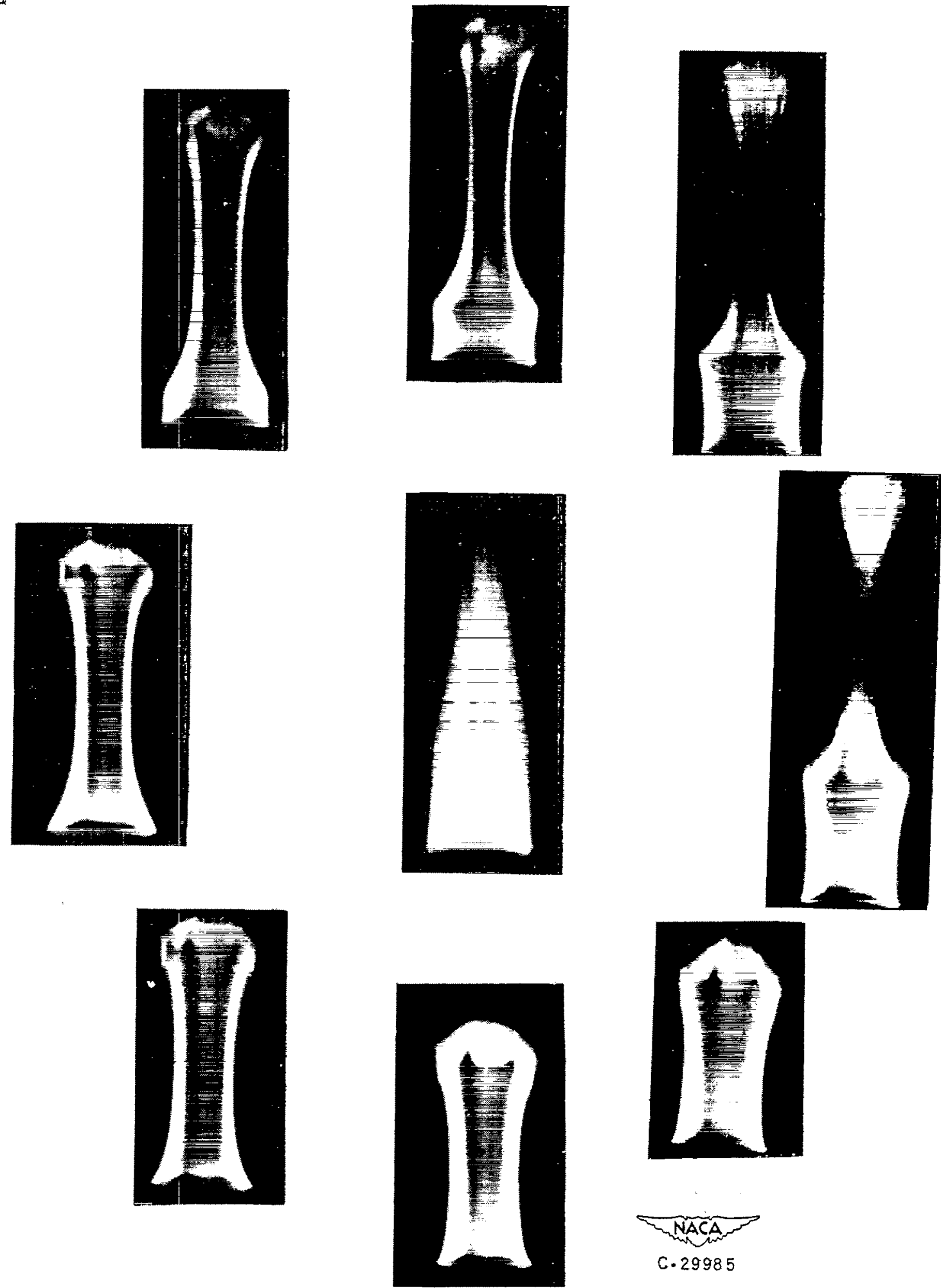

(e) Frequency, 55 cycles per second; voltage, 12.9 volts; velocity, 1.378 feet per second; fuel-air retio, 0.090 .

Figure 16. - Concluded. Sequence of flame shapes for one ç̧cle at intervals of 450. Time increases clockwlse. 

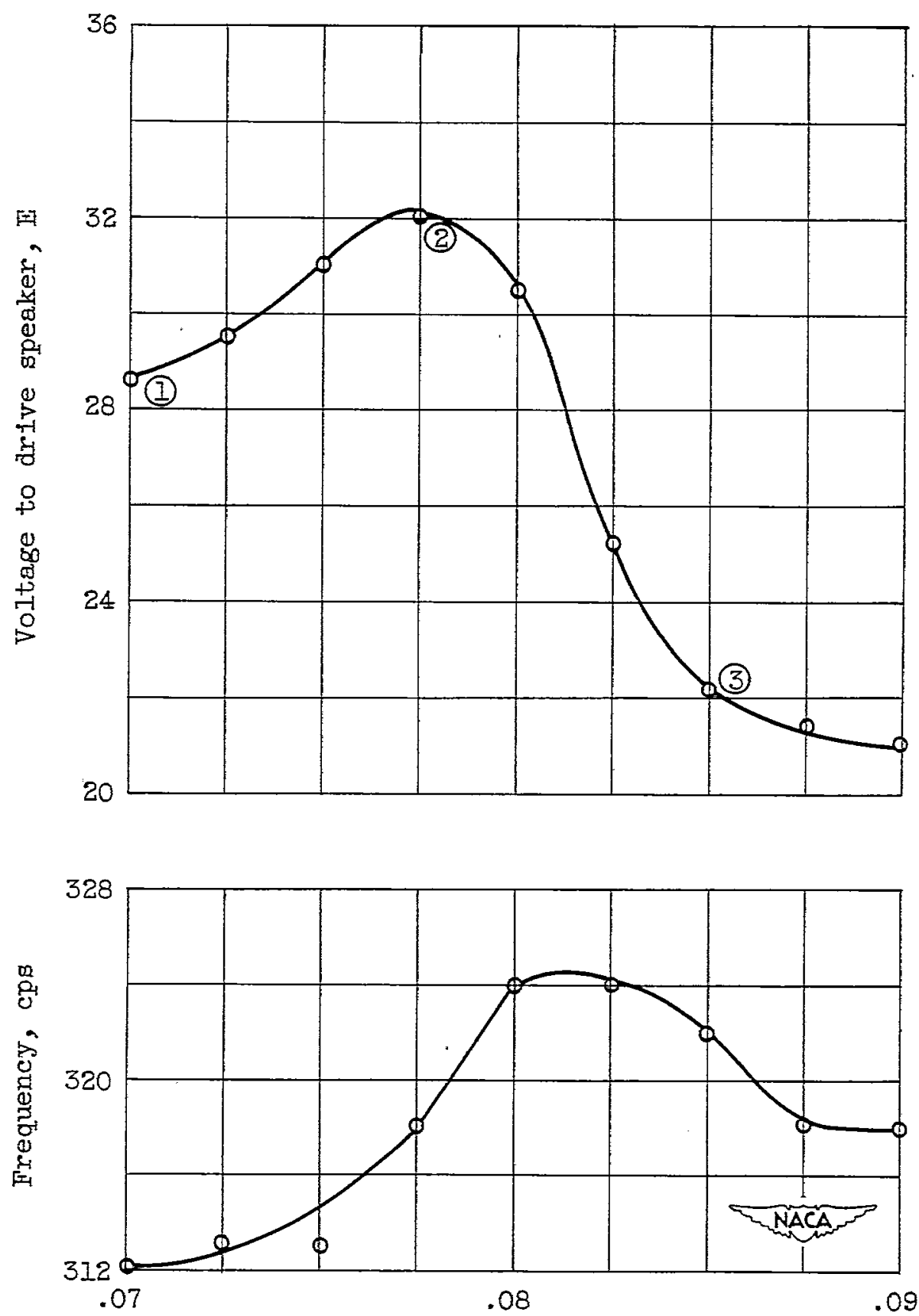

Fuel-air ratio, $f / a$

Figure 17. - Damping and frequency distribution for three-hole flame holder. Third mode. (Numbered inserts correspond to points where phase lag was determined from photographic sequences.) 


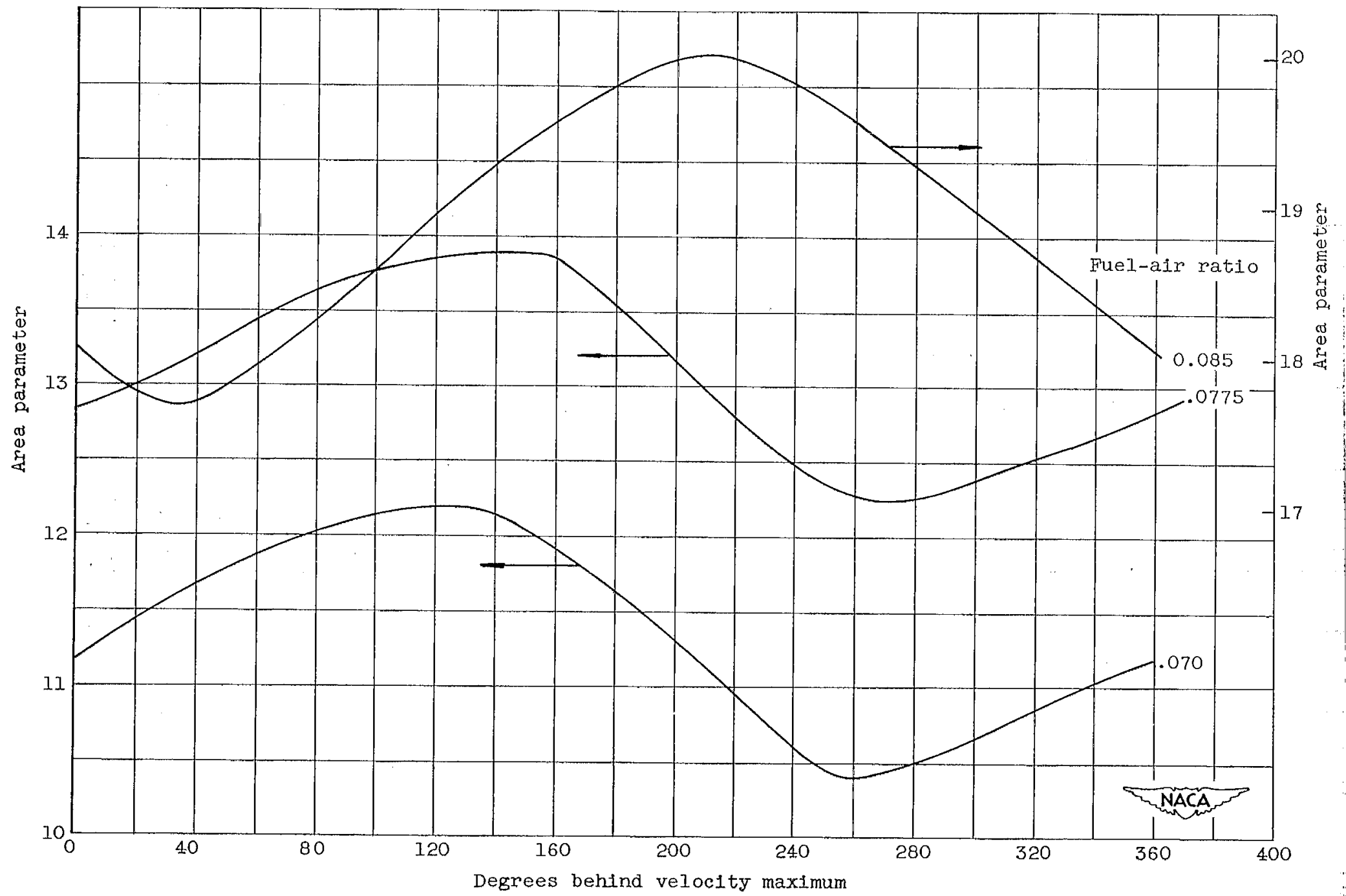

Figure 18. - Flame area as function of time for three-hole flame holder. Third mode. 